\title{
HIR-1 Mediates Response to Hypoxia-Induced Extracellular Matrix Remodeling
}

Roman Vozdek ${ }^{1}$, Yong Long ${ }^{1,2}$, Dengke K. Ma ${ }^{1, \text { * }}$

${ }^{1}$ Cardiovascular Research Institute and Department of Physiology, University of California San Francisco, San Francisco, CA 94158

${ }^{2}$ State Key Laboratory of Freshwater Ecology and Biotechnology, Institute of Hydrobiology, Chinese Academy of Sciences, Wuhan 430072, China

*Corresponding author. Email: Dengke.Ma@ucsf.edu

\section{ONE-SENTENCE SUMMARY}

A regulatory pathway for ECM homeostasis underlies adaptation to hypoxia and re-oxygenation

\author{
ABSTRACT \\ Inadequate tissue oxygen, or hypoxia, is a central concept in pathophysiology of \\ ischemic disorders and cancer. Hypoxia promotes extracellular matrix (ECM) remodeling, \\ cellular metabolic adaptation and metastasis. To determine how cells respond to hypoxia- \\ induced ECM remodeling, we performed a large-scale forward genetic screen in C. elegans. We \\ identified a previously uncharacterized receptor tyrosine kinase (RTK) named HIR-1 as a key \\ mediator in a pathway that orchestrates transcriptional responses to hypoxia-induced ECM \\ remodeling. Impaired ECM integrity caused by hypoxia or deficiency of the oxygen-dependent
}


procollagen hydroxylases, heme peroxidases or cuticular collagens activates gene expression through inhibition of HIR-1. Genetic suppressor screens identified NHR-49 and MDT-15 as transcriptional regulators downstream of HIR-1. Cellular responses through HIR-1 maintain ECM homeostasis and promote animal adaptation to severe hypoxia. We propose that $C$. elegans HIR-1 defines an unprecedented type of RTK that mediates responses to hypoxiainduced ECM remodeling by mechanisms that are likely conserved in other organisms.

\section{INTRODUCTION}

Oxygen is essential for aerobic metabolism of life. Varying oxygen levels occur in natural environments and in tissues of living organisms, eliciting highly orchestrated organismic and cellular responses to maintain proper metabolic and physiological homeostasis. For example, mammals adjust pulmonary ventilation and blood circulation to improve oxygen delivery to target tissues under hypoxic conditions, and the roundworm $C$. elegans can navigate for preferred oxygen levels across a gradient of ambient hypoxia and trigger rapid locomotor response upon severe hypoxia and restoration of oxygen (1-3). Hypoxia is also a common pathophysiological condition in human disorders characterized by a low supply of oxygen, including myocardial ischemia, stroke and tumorigenesis (4). Pathological hypoxic conditions can lead to tissue necrosis and fibrosis, degeneration, inflammation and tumor metastasis, driving disease progression and leading to organismic mortality (5).

Animals respond to chronic hypoxia through evolutionarily conserved molecular pathways and cellular mechanisms that regulate gene expression and reprogram metabolism $(4,6,7)$. The hypoxia inducible factor (HIF) is a master transcriptional regulator of hypoxic responses. Genetic studies of $C$. elegans led to the discovery of the evolutionarily conserved family of HIF hydroxylases (EGL-9 in C. elegans and EGLN2 in humans) that link oxygensensing to HIF-1 activation and transcriptional responses to hypoxia $(8,9)$. Hydroxylated HIF 
under normoxia is recognized by the Von Hippel-Lindau tumor suppressor and targeted for proteasomal degradation whereas hypoxia causes impaired HIF hydroxylation, leading to transcriptional activation of HIF target genes (10). In C. elegans, the HIF-1 pathway mediates various physiological and behavioral responses to hypoxia $(2,6,11,12)$. The transcriptional targets of mammalian HIF include LOX, LOXL2 and LOXL4 encoding copper-dependent lysyl oxidases that promote cross-linking of ECM components for enhanced tissue stiffness, a proposed trigger of tumor metastasis (5). Hypoxia-induced ECM remodeling, in turn, activates intracellular signaling cascades to regulate cell fate, metastasis and adaptation to hypoxia $(13,14)$. How cells sense and respond to hypoxia-induced ECM remodeling remains undefined.

Collagens are major integral components of ECM that are extensively modified by oxygen-dependent enzymes including lysyl oxidases, lysyl hydroxylases and prolyl hydroxylases. Beyond HIF dependent mechanisms, it is unknown whether hypoxia can alter ECM integrity directly by impairing oxygen-dependent collagen modification and how ECM remodeling might elicit subsequent HIF-independent gene regulation. In mammalian cells, VEGF (vascular endothelial growth factor) can be induced by hypoxia through both HIFdependent and HIF-independent mechanisms (15). Various protein kinases and transcription factors other than HIF respond to hypoxia in a HIF-independent manner to modulate transcriptional responses $(16,17)$. Mitogen activated protein kinases or integrin-linked protein kinases can transduce remodeled ECM signals to influence cell fate and resistance to hypoxia through transcriptional regulation $(13,14)$. C. elegans has also been extensively used to investigate HIF-independent responses that mediate physiological and behavioral adaptation to severe hypoxia (18-28). Nonetheless, the precise roles and cellular mechanisms of HIFindependent transcriptional response to hypoxia-induced ECM remodeling in physiology and diseases remain poorly understood. 
We sought to identify new genes and pathways that transduce hypoxic signals to gene regulation independently of HIF-1. We generated $C$. elegans transgenic animals carrying GFP reporters that were robustly activated by hypoxia even in HIF-1-deficient animals. We performed large-scale forward genetic screens to isolate mutants with constitutively activated reporters under normoxia and performed suppressor screens to identify hypoxia-regulated transcription factors. We found that exposure to hypoxia resulted in the remodeling of ECM, which in turn triggers intracellular transcriptional responses by inhibiting a cell transmembrane RTK that we named HIR-1 (ㅂypoxia Inhibited Receptor tyrosine kinase).

\section{RESULTS}

\section{comt-5p::GFP is a robust live reporter induced by hypoxia independently of HIF-1}

To identify genes and pathways that mediate HIF-1-independent transcriptional response by hypoxia, we used RNA-seq to compare transcriptomes of wild-type (WT) $C$. elegans under normoxia ( $21 \%$ oxygen) or severe hypoxia (nearly $0 \%$ oxygen) for 2 hours, and egl-9 null mutants (in which HIF-1 is constitutively activated) under normoxia. We identified 72 genes that showed increased expression after hypoxia in WT animals but not in egl-9 null mutants (Supplementary Fig. S1A). We generated transgenic animals with green fluorescent protein (GFP) driven by the promoters of these genes and focused on one of the reporters constructed, dmals1, that showed robust induction of GFP in the hypodermal and intestinal cells within 24 hours in $0.5 \% \mathrm{O}_{2}$ (Fig. 1A and 1B). dmals1 carries GFP driven by the promoter of comt-5, which encodes a predicted catechol-O-methyltransferase (Fig. 1C). We crossed animals carrying the dmals1 transgene with egl-9(sa307) or hif-1(ia04) loss-of-function (LOF) deletion mutants and, as expected, we did not observe activation of comt-5p::GFP in egl-9 mutants, whereas both WT and hif-1 mutants exhibited increased expression of comt-5p::GFP by hypoxia (Fig. 1A and 1D). 
To verify that endogenous comt-5 was induced by hypoxia independently of hif-1, we quantified its expression by quantitative PCR (qRT-PCR) and found that comt-5 expression was increased by hypoxia in both WT and hif-1(ia04) mutants (Fig. 1F). Other stress conditions that mimic hypoxia in activating HIF-1, including exposure of animals to $\mathrm{CoCl}_{2}, \mathrm{H}_{2} \mathrm{O}_{2}, \mathrm{CN}^{-}$or $\mathrm{H}_{2} \mathrm{~S}$, did not induce the comt-5p::GFP reporter or increase endogenous comt-5 expression (Fig. 1E). These results identify comt-5 as a specific and robust reporter gene that is activated by hypoxia independently of EGL-9 and HIF-1.

\section{HIR-1 is a cell-autonomous regulator of comt-5 that mediates hypoxia signaling}

To identify genes and pathways mediating HIF-independent regulation of dmals1, we performed large-scale forward genetic screens using ethyl methanesulfonate (EMS)-induced mutagenesis and isolated many mutants with constitutively activated comt-5p::GFP reporters even under normoxia (Table 1). Genetic linkage analysis together with whole-genome sequencing and subsequent RNA interference (RNAi) of candidate genes identified dma51 as an allele of the previously uncharacterized gene C24G6.2 (hir-1), which encodes a predicted transmembrane protein belonging to the RTK (InterPro scan) superfamily (29) (Fig. 2D). dma51 causes a LOF nonsense mutation W551Stop in HIR-1. We used CRISPR to generate a wholegene deletion allele dma101 for hir-1, which also showed constitutive comt-5p::GFP induction. A similar phenotype was also observed in mutants with partial in-frame deletion (tm3911) or an out-of-frame deletion (tm4098) that covers the exons encoding the intracellular domain of HIR-1 (Fig. 2A and 2B). A transcriptional reporter hir-1p::GFP revealed ubiquitous expression of hir-1 that was especially strong in the pharynx, hypodermal and seam cells in all developmental stages (Fig. 2E). To determine whether hir-1 regulates comt-5p::GFP cell-autonomously in the hypoderm, where comt-5p::GFP is induced by hypoxia, we generated transgenic animals with hypodermal-specific expression of hir-1(+) driven by the $d p y-7$ promoter. We observed that transgenic dpy-7p::hir-1(+) extrachromosomal arrays rescued the hir-1(LOF)-induced 
hypodermal activation of comt-5p::GFP (Fig. 2F). To rule out a possibility that hir-1 works cell non-autonomously, we generated transgenic animals with neuronal-specific expression of hir$1(+)$ driven by the pan-neuronal promoter ric-19p. We did not observe rescue of the hir-1(-)induced activation of comt-5p::GFP (Fig. S2). These results identify hir-1 as a cell-autonomous negative regulator of comt-5p::GFP.

Because hir-1 inactivation phenocopied exposure to hypoxia in comt-5p::GFP activation, we wondered whether hypoxia directly inhibits HIR-1. We generated a translational GFP reporter of HIR-1 (Fig. 2D) and observed decreased levels of full-length SDS-soluble HIR1::GFP in hypoxia-treated animals (Fig. $2 \mathrm{H}$ ). In contrast, a range of partial-length HIR-1::GFP species increased in abundance, suggesting that hypoxia leads to HIR-1 proteolytic processing (Fig. $2 \mathrm{H}$ ). Moreover, hypoxia increased the abundance of HIR-1::GFP in the protein pool that did not migrate in the SDS-PAGE, suggesting partial insolubility of HIR-1 induced by hypoxia (Fig. S2). By direct imaging of HIR-1::GFP, we observed that hypoxia induced formation of HIR$1:: G F P$ foci without affecting the transgenic co-injection marker unc-54p::mCherry in the pharyngeal muscles (Fig. 2G). These data indicate that hypoxia regulates HIR-1::GFP proteins and thereby control gene expression of comt-5 downstream of HIR-1.

\section{Inactivation of genes essential for extracellular matrix integrity mimics hypoxia and the}

\section{hir-1(-)-induced transcriptional response}

By genetic linkage mapping and whole-genome sequencing, we identified additional genes defined by other mutants isolated from EMS screens with constitutive comt-5p::GFP expression. dma52 is a Q4R mutation in dpy-2. dma22 is a G162R mutation in dpy-3. dma53 defines a previously uncharacterized gene C46A5.4, named as perl-1, which encodes a heme peroxidases-like-1 (Table 1, Fig. 3A and 3B). RNAi directed against $d p y-2, d p y-3$ or perl-1 or partial deletion alleles of these genes phenocopied EMS-derived mutations in activating constitutive expression of comt-5p::GFP under normoxia. Notably, both $d p y-2$ and $d p y-3$ encode 
cuticle collagens (30) (Fig. 3C) while perl-1 is paralogous to mlt-7, which encodes a heme peroxidase required for proper crosslinking of ECM collagens (31). Both $d p y-3$ and $d p y-2$ are expressed in hypoderm and their LOF mutations, including EMS-derived alleles, resulted in abnormal animal morphology (dumpy phenotype) and mimicked hypoxia-induced comt-5p::GFP activation (Fig. 3A). We performed 8 independent genetic screens of over 100,000 haploid genomes and observed several comt-5p::GFP activating mutants that are unable to propagate and these mutants commonly exhibited cuticle morphological defects, such as blistering or dumpy phenotypes. We used RNAi to knock-down additional genes directly responsible for cuticle biosynthesis and observed that RNAi against cuticular collagens (dpy-7, dpy-10, bli-6), collagenase inhibitor (bli-5), another heme peroxidase ( $m / t-7)$, dual oxidase complex (bli-3 and tsp-15), subtilisin-like protease (bli-4) or thioredoxin (dpy-11) activated comt-5p::GFP in the hypoderm (Table S1, Fig. S3A). Cuticle biosynthesis in the hypoderm is essential for molting and several cuticular genes are regulated by the molting-controlling transcription factors including HBL-1, NHR-23 and NHR-25 (32). RNAi against these transcription factor genes resulted in molting defects accompanied by activation of comt-5p::GFP (Table S1, Fig. S3A). These results indicate that impaired hypodermal ECM integrity, which results in dumpy or blistering morphological defects, activates comt-5p::GFP.

\section{Hypoxic stress results in the remodeling of ECM and cuticle disintegration}

Since impaired hypodermal ECM integrity increased expression of comt-5p::GFP we wondered whether hypoxia, which also leads to activation of comt-5p::GFP, leads to the remodeling of hypodermal ECM. To test whether collagens proximal to the hypodermal plasma membrane are directly affected by hypoxia, we generated transgenic animals with FLAG-tagged collagen DPY-3. By Western blot analysis we found that the molecular weight of DPY-3::FLAG was markedly altered in animals exposed to hypoxia for 24 hours (Fig. 3D), indicative of altered DPY-3::FLAG cross-linking. In contrast, another cuticle collagen COL-19 did not exhibit altered 
molecular weight after exposure to hypoxia or genetic conditions increasing expression of comt5p::GFP, including RNAi against perl-1 and $d p y$-3 (Fig. S3B). Translational reporter COL-19GFP normally localizes to the circumferential annular rings and longitudinal alae of the adult exoskeleton and genetic conditions activating comt-5p::GFP lead to disorganization of COL-19GFP (33). We examined COL-19::GFP in WT animals exposed to hypoxia for 24 hours (nearly $0 \% \mathrm{O}_{2}$ ) and observed disorganized COL-19::GFP distribution proximal to the alae (Fig. 3E). Cuticle biosynthesis begins in the endoplasmic reticulum (ER) lumen when collagens are hydroxylated by prolyl and lysyl hydroxylases allowing its trimerization, glycosylation and secretion into ECM $(34,35)$. Although there are four ER procollagen hydroxylases in $C$. elegans (dpy-18, phy-2, phy-3 or phy-4), which are partially genetically redundant (36), the hypomorphic allele of $d p y-18$ caused disorganization of the COL-19::GFP, which was similar to the pattern observed in worms exposed to hypoxia. We also observed cuticle disorganization in animals with inactivated let-268, the sole $C$. elegans lysyl hydroxylase, and perl-1 whereas inactivated dpy-3 resulted in altered GFP pattern characterized by a complete loss of the cuticular furrows (Fig. 3E). Interestingly, LET-268 activity appears to be limited to collagen type IV localized in the basement membrane but not cuticular collagens $(37,38)$. We performed RNAi against let-268 and observed larval arrest with comt-5p::GFP activation (Table S1, Fig. 3A). On the other hand, the RNAi against emb-9 encoding collagen type IV did not increase expression of the comt5p::GFP (Fig. 3A). The inactivation of let-268 leads to accumulation of the collagen type IV in the ER (38) suggesting that inactivation of let-268 activates comt-5p::GFP indirectly likely through affecting maturation of ECM proteins in the ER. These data indicate that insufficient cuticular collagen modification including hydroxylation mimics hypoxia or hir-1-induced comt5p::GFP activation in C. elegans.

In addition, WT animals exposed to hypoxia for 24 hours (nearly $0 \% \mathrm{O}_{2}$ ), perl-1, dpy-3 or dpy-18 mutants or let-268 knockdowns by RNAi exhibited exacerbated sensitivity to osmotic stress, characteristic of mutants with disrupted cuticle integrity (39), and is likely caused by 
increased cuticle permeability to water (Fig. S3C). Furthermore, direct permeability assays with the cuticle impermeable dye Hoechst 22358 (40) revealed intercalation of the dye in the nuclei only in perl-1 and dpy-3 mutants, let-268 knockdowns and hypoxia-treated WT animals but not WT animals under normoxia (Fig. S3D). These data provide multiple independent lines of evidence that cuticle in animals exposed to hypoxia exhibits altered integrity characterized by increased permeability.

\section{NHR-49 and MDT-15 mediate comt-5 transcriptional response to hypoxia}

To identify specific transcription factors that drive comt-5p::GFP expression in response to hypoxia and ECM remodeling, we sought second-site suppressor mutations of the most penetrant comt-5p::GFP-activating mutation dma11 isolated from EMS screens (Table 1). The gene defined by dma11 remains as yet unidentified. Nonetheless, we isolated two independent suppressing alleles dma53 and dma54 and used linkage analysis and RNAi phenocopying to identify them as mutations of $m d t-15$ and $n h r-49$, respectively. dma53 is a nonsense mutation leading to a premature stop codon in $m d t-15$, and dma54 is a missense mutation G33R in $n h r-$ 49 (Fig. 4B). NHR-49 and MDT-15 are transcriptional regulators that physically interact to regulate lipid homeostasis (41). Protein sequence analysis of orthologous nuclear hormone receptors revealed that Gly ${ }^{33}$ is in the conserved DNA binding domain among all examined sequences, including the vertebrate orthologue HNF4 (Fig. 4D). To verify that LOF of $n h r-49$ also suppressed hir-1-induced comt-5p::GFP activation, we crossed the $n h r-49$ null allele nr2041 with hir-1 mutants and found that the hir-1; nhr-49 double mutants exhibited suppressed comt-5p::GFP. Moreover, hypoxia did not activate comt-5p::GFP in nhr-49 or mdt-15 null mutants whereas the gain-of-function mutations $n h r-49(e t 7)$ and $m d t-15($ et14) showed constitutively activated comt-5p::GFP under normoxia (Fig. 4A). Western blot and qRT-PCR analysis confirmed the requirement for NHR-49 and MDT-15 in the activation of comt-5p::GFP 
in hir-1 mutants (Fig. 4C). qRT-PCR analysis also confirmed that both $n h r-49$ and $m d t-15$ were required for hypoxic induction of comt-5 (Fig. 4E). These data indicate that transcriptional activation of comt-5 by hypoxia or LOF of HIR-1 requires NHR-49 and MDT-15.

NHR-49 and MDT-15 orchestrate lipid homeostasis in C. elegans (42) and mediate response to changes in lipid metabolic cues and temperature $(11,42)$. We generated a transgenic strain expressing $n h r-49::$ Venus under the ubiquitous $r p /-28$ promoter. We did not observe altered pattern of the subcellular localization of NHR-49::Venus after hypoxia, suggesting that NHR-49 is not regulated at the level of nuclear translocation. In addition, we observed that comt-5p::GFP induction by hir-1 inactivation was limited to adulthood and increased with age, whereas $n h r-49$ gain-of-function mutation or hypoxia activated comt5p::GFP in all developmental stages (Fig. S4A). These results support that NHR-49 is essential for HIR-1-dependent transcriptional response but plays broader roles than HIR-1.

\section{Mechanisms of HIR-1 regulation by hypoxia-induced ECM remodeling}

The genetic evidence connecting hypoxia, ECM integrity and hir-1 suggests that exposure to hypoxia induces remodeling of the ECM, leading to inhibition of HIR-1 and subsequent activation of comt-5p::GFP. The intracellular domain of HIR-1 is structurally similar to the proto-oncogene receptor RET and fibronectin growth factor (FGF) receptors, with conserved catalytic sites essential for autophosphorylation (Fig. 5A). We wondered whether the HIR-1 kinase activity could be inhibited by depletion of ATP due to hypoxia. We exposed animals to rotenone, an inhibitor of the oxidative phosphorylation, but did not observe increased expression of comt-5p::GFP. The extracellular domain of HIR-1 resembles immunoglobulin-like fold (a.a. 80-446) including fibronectin type III-like fold (a.a. 84-170), domains common in many receptors previously identified as interacting with ECM proteins $(43,44)$. FGF4 is a ligand implicated in mediating ECM sensing to regulate trophoblast stem cell fate (13). Inactivation of C. elegans FGF-encoding gene let-756 by RNAi led to constitutive activation of comt-5p::GFP 
under normoxia, phenocopying hir-1 LOF mutants (Fig. 5B). We generated an HA-tagged translational reporter of let-756 and observed hypoxia-induced change in LET-756 molecular weights (Fig. 5C). These findings indicate co-regulation of HIR-1 and LET-756, supporting the notion that HIR-1 acts with FGF-like proteins to sense ECM remodeling upon hypoxia, which would attenuate binding of FGF-like ligands and thereby inactivating HIR-1.

\section{HIR-1 regulates a broad genetic program controlling ECM homeostasis and protects against severe hypoxic stress}

To systematically identify genes differentially regulated by hir-1 in relation to hypoxia, we performed RNA-seq analysis of hir-1 mutants and compared transcriptomes of WT animals under normoxia or hypoxia and hir-1 mutants (Fig. S1B, S5A and S5B). Expression of genes that regulate comt-5p::GFP, including $d p y-2, d p y-3$, perl-1, was increased by both hypoxia and hir-1 inactivation (Fig. S2A). We used qRT-PCR to quantify the expression levels of genes encoding collagens, procollagen hydroxylases, peroxidases and transcription factors mediating molting processes in hir-1, hir-1; nhr-49 and hir-1; hif-1 mutants. All examined genes showed increased expression in hir-1 and hir-1; hif-1 mutants and decreased expression in hir-1; nhr-49 mutants (Fig. S2B). Furthermore, we found that genes involved in molting and cuticle integrity showed increased expression in hir-1 mutants, including members of collagen-encoding dpy genes whose inactivation can cause the Dumpy phenotype (Data File S1). Several collagenencoding non-Dumpy genes, including col-17 and col-41, were suppressed (Fig. S5B). Collagens are the main structural components of the ECM and cuticle, and the epicuticle contains lipids that regulate its permeability (46). We found that hir-1 mutants exhibited increased expression of genes involved in lipid metabolism, including acs-2, a target gene of NHR-49 and key regulator of fatty acid homeostasis (42) (Fig. S5B). LOF of hir-1 led to disrupted cuticle integrity, supporting that hir-1 mutants exhibit more permeable cuticles (Fig. 6B and $6 \mathrm{C}$, and S3F). The cuticle defects of hir-1 mutants was not fully rescued by $n h r-49$ LOF, 
indicating that defects in ECM integrity of hir-1 mutants under normoxia involves abnormal activation of additional unidentified transcription factors (Fig. S4B).

We next tested whether the genetic program mediated by HIR-1 signaling pathway was essential for resistance to severe hypoxic stress. We exposed 1-day old WT and hir-1(dma101) mutants to anoxia for 40 hours and subsequently compared their locomotion behavior after reoxygenation. All tested animals were in suspended animation-like state unable to respond to external stimuli (mechanic touch or UV light) during anoxia and immediately after reoxygenation, which was followed by behavioral recovery in crawling (defined as displacement) and pharyngeal pumping. We found that the displacement of re-oxygenated WT animals gradually recovered, whereas hir-1 mutants had markedly severe locomotion defects that did not improve even after 24 hours of re-oxygenation and culminated in enhanced rates of organismic death (Fig. 6D-F). These results indicate that HIR-1 plays key roles to control a broad genetic program that guards against maladaptive cuticle collagen and ECM homeostasis for animal survival upon exposure to severe hypoxia (Fig. 6G).

\section{DISCUSSION}

How cells in multicellular organisms sense hypoxia-remodeled ECM to promote cuticle homeostasis and facilitate animal adaptation to severe hypoxia is unknown. From genetic screens, we identified HIR-1 as a key mediator of the transcriptional response to hypoxiainduced ECM remodeling $(47,48)$. Altered expression pattern of GFP-tagged HIR-1 in animals exposed to hypoxia suggests that HIR-1 is directly regulated by hypoxia-induced ECM remodeling. The observed HIR-1::GFP foci are reminiscent of aggregation and proteolytic processing, a previously observed effect of hypoxia on other proteins in $C$. elegans $(27,28)$. We do not exclude a possibility of receptor clustering, internalization or block of the membrane trafficking that would affect activity of HIR-1 or its interacting partners. HIR-1 does not possess the discoidin domain, which is responsible for interaction with collagens to sense ECM 
remodeling (45). Our genetic evidence suggests that LET-756 homologous to mammalian FGFs is a likely HIR-1 ligand, whose binding to HIR-1 might be modulated by hypoxia-induced ECM remodeling. How precisely HIR-1 is regulated by ECM remodeling in coordination with its ligand and, in turn, transduces intracellular signaling for ECM homeostasis awaits further studies.

The integrity and composition of ECM are sensitive to oxygen availability due to oxygendependent enzymatic activities of prolyl and lysyl hydroxylases in the ER as well as dual oxidases in the extracellular space $(31,49)$. We found that procollagen hydroxylase or dual oxidase inactivation by RNAi phenocopied hypoxia-induced activation of comt-5p::GFP. LOF phenotypes of these oxygen-dependent enzymes suggest that they likely mediate hypoxic response directly, whereas their HIF-induced increase in expression might constitute a homeostatic response to eventually restore their activity in the cell. Depending on the tissuespecific range of oxygen levels to which these enzymes are sensitive, procollagen hydroxylases and extracellular oxidases likely act as cellular oxygen sensors that mediate responses to varying degrees of hypoxia. We propose that such oxygen sensing in the ER or extracellular space acts in parallel to cytosolic EGLN oxygen sensors to mediate HIF-independent transcriptional programs to induce ECM remodeling and inhibit HIR-1 to maintain ECM homeostasis and animal survival under severe hypoxic conditions.

Hypoxia-induced ECM remodeling occurs in cultured tumor cells (5) and ECM surrounding bone marrow tumor cells exhibit increased stiffness that triggers metastasis (50). Increased stiffness of the ECM due to enhanced crosslinking of ECM proteins is mediated by increased expression of lysyl hydroxylase and lysyl oxidase and increased collagen deposition $(5,51,52)$. Similarly, we also found hypoxia increased the expression of genes encoding procollagen lysyl oxidase, ER hydroxylases, and collagens in C. elegans, indicating conserved transcriptional responses that promote homeostatic ECM remodeling, which may have been coopted by tumors to facilitate hypoxic survival and metastasis. ECM remodeling during aging 
through differential regulation of collagen-encoding genes contributes to extension of $C$. elegans longevity (53), consistent with the notion that homeostatic ECM remodeling mediated by the HIR-1 pathway promotes cellular and organismic resistance to hypoxic stresses.

We found that proper HIR-1 regulation is important for $C$. elegans adaptation to hypoxic stress and re-oxygenation while its downstream transcriptional effectors include not only ECM components but also a non-ECM gene comt-5. comt-5 is predicted to encode a catecholaminedegrading enzyme, and its upregulation by hypoxia likely help decrease catecholamine levels to alleviate the toxicity of their oxidized forms under hypoxia, while its constitutive expression in hir-1 mutants under normoxia is likely detrimental. Dopamine targets peripheral tissues to maintain xenobiotic stress resistance in C. elegans (54), however, whether upregulation of comt-5 is required for proper resistance to hypoxic and/or xenobiotic stress awaits further studies. Although we used primarily comt-5::GFP reporters for gene and pathway discoveries, transcriptomic analysis revealed that $\mathrm{HIR}-1$ regulates a broad genetic program responsible for remodeling of ECM, altering cuticle integrity and reprograming lipid metabolism.

Our findings suggest that hir-1- and nhr-49-mediated reprogramming of lipid metabolism likely also contributes to hypoxic survival in addition to ECM homeostasis. Hypoxic regulation of genes involved in cuticular lipid synthesis can promote cuticle permeability and adaptation to hypoxia in Arabidopsis thaliana (55). Lipid metabolic reprogramming also contributes to tumor development and hypoxic resistance in mammals (56). Upstream of NHR-49, the regulatory axis from oxygen, ER hydroxylases, and collagen to RTKs appears to share features common in both $C$. elegans and humans. We thus propose that the HIR-1 pathway is evolutionarily conserved to mediate cellular response to hypoxia and ECM remodeling in diverse organisms. Numerous human RTKs, including FGFRs, have been implicated in driving tumor survival and metastasis $(57,58)$, and ECM remodeling is essential for the progression of cancer, tissue fibrosis and many other diseases involving ECM dysregulation (59). Given the central role of 
HIR-1 in mediating cellular response through ECM remodeling to promote resistance to hypoxia, the human counterpart of HIR-1 may, once verified, be a promising therapeutic target for solid tumors that survive severe hypoxia and metastasize through ECM remodeling.

\section{MATERIALS AND METHODS}

\section{C. elegans strains}

Animals were maintained under standard procedure with nematode growth media (NGM) plates unless otherwise stated. Bristol strain N2 was used as wild type and Hawaiian strain CB4856 was used for the linkage analysis of the mutants $(60,61)$. hir-1 null alleles were generated by CRISPR to induce double stranded breaks and subsequent non-homologous end joining caused a deletion of hir-1. Feeding RNAi was performed as previously described (62).

Transgenic strains were generated by germline transformation as described (63). Transgenic constructs were co-injected with dominant unc-54p::mCherry or myo-2::mCherry markers, and stable extrachromosomal lines of mCherry+ animals were established. Transgenic strains used were DMS24 (dmals1), DMS23 (dmals1;hif-1(ia04)), DMS22 (dmals1;egl-9(sa307)), DMS176 (dmals1; hir-1(dma51)), DMS290 (dmals1; hir-1(dma101)), DMS492 (dmals1;hir-1(tm3911)), DMS311 (dmals1;hir-1(tm4098)), DMS634 (dmals1;hir-1(dma101);nhr-49(nr2041)), DMS290 (dmals1;hir-1(dma101), DMS99 (dmals1;dma11), DMS181(dmals1;dma11;mdt-15(dma54)), DMS182 (dmals1;dma11;nhr-49(dma55)), DMS283 (dmals1;mdt-15(et14)), DMS127 (dmals1;dpy-3(dma22)), DMS177 (dmals1;dpy-2(dma52)), DMS180 (dmals1;perl-1(dma53)), DMS582 (qyls44[emb-9::mCherry]), DMS696 (dmals1;perl-1(dma236)), DMS636 (dmals1; hir1(dma101); dmaEx113[dpy-7::hir-1(+); myo-2::mCherry]), DMS665 (dmaEx121[rpl-28p::hir1::SignalPeptide::gfp::hir-1; myo-2p::mCherry]), TP12 (kals12[col-19::GFP]).

\section{Genetic screens}


Stereo-epifluorescence dissecting microscope (Nikon SMZ18) was used to isolate mutants with constitutive expression of comt-5p::GFP reporters after ethyl methanesulfonate (EMS)-induced mutagenesis, as described previously $(2,11)$. Mutants were mapped genetically by single nucleotide polymorphisms-based linkage analysis using the Hawaiian C. elegans strain CB4856 and then were sequenced by whole-genome sequencing to obtain lists of candidate genes. Genes with putative causal mutations were verified by RNA interference that can phenocopy mutants and the causality of mutation was subsequently confirmed by transformation rescue of mutants with wild-type alleles as transgenes.

\section{Environmental stress assays}

Hypoxia chamber with ProOx110 oxygen controller (Biospherix) was used for hypoxia stress with $0.5 \%-21 \%$ oxygen concentrations. Hypoxia incubator chamber (Applied StemCell) with constant nitrogen flow delivering was used for achieving severe hypoxia with nearly $0 \%$ oxygen (anoxia stress). In hydrogen peroxide stress assay, animals were placed on the plate with 10 $\mathrm{mM}$ peroxide in the NGM and observed in the 1-48-hour interval for GFP activation. For determining effects of HIF-activating compounds on comt-5p::GFP, $5 \mathrm{mM} \mathrm{CoCl}_{2}, 5 \mathrm{mM} \mathrm{KCN}$ containing NGM plates were used. For assaying hydrogen sulfide, $0.1 \mathrm{mg}$ of NaHS powder was placed onto NGM agar plate (10 $\mathrm{ml}$ of agar) with sealed lid by parafilm to prevent leaking of the released $\mathrm{H}_{2} \mathrm{~S}$ gas. Animals were subsequently screened for the GFP induction in the 1-48-hour interval, and for the qRT-PCR analysis collected after 2 hours of exposure.

\section{Western Blot analysis}

Animals were lysed in the Laemmli sample buffer (BioRad) with reducing agent betamercaptoethanol followed by boiling the samples for 10 minutes. The worm lysates were separated by SDS-PAGE and subsequently detected by GFP Goat polyclonal antibody (Fisher 
scientific - AF424) with Histone H3 antibody (AbCam - ab1791) as a loading control. For mCherry-tagged EMB-9, we used mCherry antibody 16D7 (Thermo Fisher Scientific M11217).

\section{Sample and library preparation for RNA sequencing}

All C. elegans strains (N2(WT), egl-9(sa307), egl-9(sa307); hif-1(ia04), hir-1(tm4098)), were maintained at $20^{\circ} \mathrm{C}$ prior RNA extraction. For anoxia stress, we placed N2 animals into hypoxia incubator chamber (Applied StemCell) with constant nitrogen delivering for 2 hours before lysis. $1 \mu \mathrm{g}$ of total RNA from each sample was purified by RNeasy Mini Kit from Qiagen and used for sequencing library construction. The NEBNext® rRNA Depletion Kit, Agencourt RNAClean XP Beads from Beckman Coulter, NEBNext® Ultra ${ }^{\text {TM }}$ Directional RNA Library Prep Kit for Illumina $\AA$, Agencourt AMPure XP from Beckman Coulter and NEBNext Multiplex Oligos for Illumina were used for preparation of sequencing libraries, per manufacturers' instruction. The Q5 Hot Start HiFi PCR Master Mix was used for PCR enrichment of the adaptor-ligated DNA. The libraries were submitted to 100 bp paired-end high throughput sequencing using Hiseq3000 by the Center for Advanced Technology of the University of California, San Francisco.

\section{RNA-seq data analysis}

The prinseq-lite software (0.20.4) was used (64) to trim and filter raw reads. Reads longer than 30 bp together with minimum quality score $>15$ were used for subsequent analyses. The Pairfq script was used for separation of paired and single reads. Clean reads were mapped to the $C$. elegans genome using Hisat2 (2.0.5) (65) with default parameters. The number of mapped reads were counted by featureCounts (1.5.0) (66). Differential gene expression analysis was performed using the DESeq2 package (67). Adjusted P-value $\leq 0.05$ was used as the threshold to identify the differentially expressed genes. Gene ontology and KEGG pathway enrichment analyses for the differentially expressed genes were conducted using the Cytoscape plugins 
BiNGO (68) and ClueGO (69), respectively. Plots for the mapped reads were generated by IGVtools (70).

\section{Quantitative RT-PCR}

Total RNAs were isolated from animals of mixed stages, with $50 \mu$ pellet animals $(>200$ animals) resuspended in $250 \mu$ lysis buffer of Quick-RNA MiniPrep kit (Zymo Research, R1055) and subsequently lysed by TissueRuptor (Motor unit "8" for 1 min). Total RNAs were extracted following instruction (Zymo Research, R1055). $2 \mu \mathrm{g}$ RNA/sample was reverse transcribed into cDNA (BioTools, B24408). Real-time PCR was performed by using Roche LightCycler®96 and SYBR Green (Thermo Fisher Scientific, FERK1081) as a dsDNA-specific binding dye. qRT-PCR condition was set to $95^{\circ} \mathrm{C}$ for denaturation, followed by 45 cycles of $10 \mathrm{~s}$ at $95^{\circ} \mathrm{C}, 10 \mathrm{~s}$ at $60^{\circ} \mathrm{C}$, and $20 \mathrm{~s}$ at $72^{\circ} \mathrm{C}$. Melting curve analysis was performed after the final cycle to examine the specificity of primers in each reaction. Gene expression changes were calculated by $\Delta \Delta \mathrm{CT}$ method with act-3 used as reference gene. Primer sequences are in table S2.

\section{Imaging}

Animals were mounted onto $2 \%$ agarose pad with $10 \mathrm{mM}$ sodium azide and imaged with EVOS FL auto digital microscope for epifluorescence imaging or the confocal Leice SPE microscope for high-resolution col-19::GFP confocal imaging. At least 3 biological replicates ( $\geq 10$ animals for each replicate) were used for quantification of the designated phenotype.

\section{Hoechst staining}

Animals were placed into liquid drops containing $2 \mu \mathrm{g} / \mathrm{ml}$ of the Hoechst 22358 dye diluted in M9 buffer for 15 minutes. Then the animals were picked into fresh M9 drops and subsequently placed onto $2 \%$ agarose pad with $10 \mathrm{mM}$ sodium azide for imaging by the confocal Leica SPE 
microscope. At least 3 biological replicates ( $\geq 10$ animals for each group) were used for quantification of stained animals.

\section{Osmotic shock assay}

5-day old adult hermaphrodites were picked and placed into $200 \mu$ liquid drop of PCR-grade distilled water drop on plastic lid of Petri plate. Time when cuticle bursted (release of insides) was monitored by eye in 15-second intervals. 3 biological replicates (10 animals without bacteria per assay) were used for statistical analysis.

\section{Anoxia sensitivity assay}

Animals were grown at $25^{\circ} \mathrm{C}$ for two continuous generations in non-starving non-stressed conditions. 1-day old adult hermaphrodites were placed on the NGM plates into the anoxia chamber for 40 hours. Animals were subsequently screened for their paralysis/movement in the indicated time intervals. WormLab system (MBF Bioscience) was used for quantification of the displacement, moving average speed and tracking based on the mid-point position. At least 3 biological replicates (10 animals per assay) were used for statistical analysis.

\section{Statistical analysis}

Data are presented as means \pm S.D. unless otherwise specified with $p$ values calculated by unpaired Student's t-tests and one-way ANOVA.

\section{SUPPLEMENTARY MATERIALS}

Fig.S1. Venn diagrams for differentially regulated genes.

Fig.S2. RNA-seq and qRT-PCR analysis.

Fig.S3. ECM and cuticle integrity assays.

Fig.S4. HIR-1 mediates ECM homeostasis. 
Fig.S5. Volcano plot and Heat map

Table S1. Genes that regulate comt-5p::GFP expression.

Table S2. Primer sequences

Data File S1. Gene ontology and KEGG pathway analyses of genes differentially regulated in hir-1 mutants compared with wild-type animals.

\section{REFERENCES AND NOTES}

1. Chang AJ, Chronis N, Karow DS, Marletta MA, Bargmann Cl. A Distributed Chemosensory Circuit for Oxygen Preference in C. elegans. PLOS Biol. 2006 Aug 15;4(9):e274.

2. Ma DK, Vozdek R, Bhatla N, Horvitz HR. CYSL-1 interacts with the O2-sensing hydroxylase EGL-9 to promote H2S-modulated hypoxia-induced behavioral plasticity in C. elegans. Neuron. 2012 Mar 8;73(5):925-40.

3. Kumar P, Prabhakar NR. Peripheral chemoreceptors: function and plasticity of the carotid body. Compr Physiol. 2012 Jan;2(1):141-219.

4. Semenza GL. Hypoxia-inducible factors in physiology and medicine. Cell. 2012 Feb 3;148(3):399408.

5. Gilkes DM, Semenza GL, Wirtz D. Hypoxia and the extracellular matrix: drivers of tumour metastasis. Nat Rev Cancer. 2014 Jun;14(6):430-9.

6. Shen C, Powell-Coffman JA. Genetic analysis of hypoxia signaling and response in C elegans. Ann N Y Acad Sci. 2003 May;995:191-9.

7. Kaelin WG, Ratcliffe PJ. Oxygen sensing by metazoans: the central role of the HIF hydroxylase pathway. Mol Cell. 2008 May 23;30(4):393-402.

8. Trent C, Tsuing N, Horvitz HR. Egg-laying defective mutants of the nematode Caenorhabditis elegans. Genetics. 1983 Aug;104(4):619-47.

9. Epstein AC, Gleadle JM, McNeill LA, Hewitson KS, O'Rourke J, Mole DR, et al. C. elegans EGL-9 and mammalian homologs define a family of dioxygenases that regulate HIF by prolyl hydroxylation. Cell. 2001 Oct 5;107(1):43-54.

10. Semenza GL. HIF-1, O(2), and the 3 PHDs: how animal cells signal hypoxia to the nucleus. Cell. 2001 Oct 5;107(1):1-3.

11. Ma DK, Li Z, Lu AY, Sun F, Chen S, Rothe M, et al. Acyl-CoA Dehydrogenase Drives Heat Adaptation by Sequestering Fatty Acids. Cell. 2015 May 21;161(5):1152-63. 
12. Leiser SF, Miller H, Rossner R, Fletcher M, Leonard A, Primitivo M, et al. Cell nonautonomous activation of flavin-containing monooxygenase promotes longevity and health span. Science. 2015 Dec 11;350(6266):1375-8.

13. Choi HJ, Sanders TA, Tormos KV, Ameri K, Tsai JD, Park AM, et al. ECM-Dependent HIF Induction Directs Trophoblast Stem Cell Fate via LIMK1-Mediated Cytoskeletal Rearrangement. PLOS ONE. 2013 Feb 21;8(2):e56949.

14. Pang M-F, Siedlik MJ, Han S, Stallings-Mann M, Radisky DC, Nelson CM. Tissue Stiffness and Hypoxia Modulate the Integrin-Linked Kinase ILK to Control Breast Cancer Stem-like Cells. Cancer Res. 2016 Sep 15;76(18):5277-87.

15. Mizukami Y, Li J, Zhang X, Zimmer MA, Iliopoulos O, Chung DC. Hypoxia-inducible factor-1independent regulation of vascular endothelial growth factor by hypoxia in colon cancer. Cancer Res. 2004 Mar 1;64(5):1765-72.

16. Cummins EP, Taylor CT. Hypoxia-responsive transcription factors. Pflugers Arch. 2005 Sep;450(6):363-71.

17. Lee DC, Sohn HA, Park Z-Y, Oh S, Kang YK, Lee K-M, et al. A lactate-induced response to hypoxia. Cell. 2015 Apr 23;161(3):595-609.

18. Park EC, Ghose P, Shao Z, Ye Q, Kang L, Xu XZS, et al. Hypoxia regulates glutamate receptor trafficking through an HIF-independent mechanism. EMBO J. 2012 Mar 21;31(6):1379-93.

19. Ladage ML, King SD, Burks DJ, Quan DL, Garcia AM, Azad RK, et al. Glucose or Altered Ceramide Biosynthesis Mediate Oxygen Deprivation Sensitivity Through Novel Pathways Revealed by Transcriptome Analysis in Caenorhabditis elegans. G3 Bethesda Md. 2016 Oct 13;6(10):3149-60.

20. Ghose P, Park EC, Tabakin A, Salazar-Vasquez N, Rongo C. Anoxia-reoxygenation regulates mitochondrial dynamics through the hypoxia response pathway, SKN-1/Nrf, and stomatin-like protein STL-1/SLP-2. PLoS Genet. 2013;9(12):e1004063.

21. Wouters BG, Koritzinsky M. Hypoxia signalling through mTOR and the unfolded protein response in cancer. Nat Rev Cancer. 2008 Nov;8(11):851-64.

22. Peña S, Sherman T, Brookes PS, Nehrke K. The Mitochondrial Unfolded Protein Response Protects against Anoxia in Caenorhabditis elegans. PloS One. 2016;11(7):e0159989.

23. Padmanabha D, Padilla PA, You Y-J, Baker KD. A HIF-Independent Mediator of Transcriptional Responses to Oxygen Deprivation in Caenorhabditis elegans. Genetics. 2015 Mar;199(3):739-48.

24. Lee J, Lee J. Hypoxia-inducible Factor-1 (HIF-1)-independent hypoxia response of the small heat shock protein hsp-16.1 gene regulated by chromatin-remodeling factors in the nematode Caenorhabditis elegans. J Biol Chem. 2013 Jan 18;288(3):1582-9.

25. Padilla PA, Nystul TG, Zager RA, Johnson ACM, Roth MB. Dephosphorylation of cell cycle-regulated proteins correlates with anoxia-induced suspended animation in Caenorhabditis elegans. Mol Biol Cell. 2002 May;13(5):1473-83. 
26. Miller DL, Roth MB. C. elegans are protected from lethal hypoxia by an embryonic diapause. Curr Biol CB. 2009 Jul 28;19(14):1233-7.

27. Fawcett EM, Hoyt JM, Johnson JK, Miller DL. Hypoxia disrupts proteostasis in Caenorhabditis elegans. Aging Cell. 2015 Feb;14(1):92-101.

28. Kaufman DM, Wu X, Scott BA, Itani OA, Van Gilst MR, Bruce JE, et al. Ageing and hypoxia cause protein aggregation in mitochondria. Cell Death Differ. 2017;24(10):1730-8.

29. Finn RD, Attwood TK, Babbitt PC, Bateman A, Bork P, Bridge AJ, et al. InterPro in 2017-beyond protein family and domain annotations. Nucleic Acids Res. 2017 Jan 4;45(D1):D190-9.

30. Johnstone IL. Cuticle collagen genes: expression in Caenorhabditis elegans. Trends Genet. 2000 Jan 1;16(1):21-7.

31. Thein MC, Winter AD, Stepek G, McCormack G, Stapleton G, Johnstone IL, et al. Combined extracellular matrix cross-linking activity of the peroxidase MLT-7 and the dual oxidase BLI-3 is critical for post-embryonic viability in Caenorhabditis elegans. J Biol Chem. 2009 Jun 26;284(26):17549-63.

32. Hayes GD, Frand AR, Ruvkun G. The mir-84 and let-7 paralogous microRNA genes of Caenorhabditis elegans direct the cessation of molting via the conserved nuclear hormone receptors NHR-23 and NHR-25. Development. 2006 Dec 1;133(23):4631-41.

33. Thein MC, McCormack G, Winter AD, Johnstone IL, Shoemaker CB, Page AP. Caenorhabditis elegans exoskeleton collagen COL-19: an adult-specific marker for collagen modification and assembly, and the analysis of organismal morphology. Dev Dyn Off Publ Am Assoc Anat. 2003 Mar;226(3):523-39.

34. Johnstone IL. The cuticle of the nematode Caenorhabditis elegans: a complex collagen structure. BioEssays News Rev Mol Cell Dev Biol. 1994 Mar;16(3):171-8.

35. Prockop DJ, Kivirikko KI. Collagens: Molecular Biology, Diseases, and Potentials for Therapy. Annu Rev Biochem. 1995 Jun 1;64(1):403-34.

36. Friedman L, Higgin JJ, Moulder G, Barstead R, Raines RT, Kimble J. Prolyl 4-hydroxylase is required for viability and morphogenesis in Caenorhabditis elegans. Proc Natl Acad Sci. 2000 Apr 25;97(9):4736-41.

37. Cox GN, Kusch M, Edgar RS. Cuticle of Caenorhabditis elegans: its isolation and partial characterization. J Cell Biol. 1981 Jul;90(1):7-17.

38. Norman KR, Moerman DG. The let-268 locus of Caenorhabditis elegans encodes a procollagen lysyl hydroxylase that is essential for type IV collagen secretion. Dev Biol. 2000 Nov 15;227(2):690-705.

39. Wheeler JM, Thomas JH. Identification of a novel gene family involved in osmotic stress response in Caenorhabditis elegans. Genetics. 2006 Nov;174(3):1327-36. 

aCC-BY 4.0 International license.

40. Ward JD, Mullaney B, Schiller BJ, He LD, Petnic SE, Couillault C, et al. Defects in the C. elegans acylCoA Synthase, acs-3, and Nuclear Hormone Receptor, nhr-25, Cause Sensitivity to Distinct, but Overlapping Stresses. PLoS ONE [Internet]. 2014 Mar 20 [cited 2017 Nov 14];9(3). Available from: https://www.ncbi.nlm.nih.gov/pmc/articles/PMC3961378/

41. Taubert S, Hansen M, Van Gilst MR, Cooper SB, Yamamoto KR. The Mediator subunit MDT-15 confers metabolic adaptation to ingested material. PLoS Genet. 2008 Feb 29;4(2):e1000021.

42. Gilst MRV, Hadjivassiliou H, Jolly A, Yamamoto KR. Nuclear Hormone Receptor NHR-49 Controls Fat Consumption and Fatty Acid Composition in C. elegans. PLOS Biol. 2005 Feb 8;3(2):e53.

43. Aota S, Nagai T, Yamada KM. Characterization of regions of fibronectin besides the arginineglycine-aspartic acid sequence required for adhesive function of the cell-binding domain using sitedirected mutagenesis. J Biol Chem. 1991 Aug 25;266(24):15938-43.

44. Kim S-H, Turnbull J, Guimond S. Extracellular matrix and cell signalling: the dynamic cooperation of integrin, proteoglycan and growth factor receptor. J Endocrinol. 2011 May 1;209(2):139-51.

45. Fu H-L, Valiathan RR, Arkwright R, Sohail A, Mihai C, Kumarasiri M, et al. Discoidin Domain Receptors: Unique Receptor Tyrosine Kinases in Collagen-mediated Signaling. J Biol Chem. 2013 Mar 15;288(11):7430-7.

46. Page AP, Johnstone IL. The cuticle. WormBook Online Rev C Elegans Biol. 2007 Mar 19;1-15.

47. Moreno-Arriola E, Hafidi ME, Ortega-Cuéllar D, Carvajal K. AMP-Activated Protein Kinase Regulates Oxidative Metabolism in Caenorhabditis elegans through the NHR-49 and MDT-15 Transcriptional Regulators. PLOS ONE. 2016 Jan 29;11(1):e0148089.

48. Mennerich D, Dimova EY, Kietzmann T. Direct phosphorylation events involved in HIF- $\alpha$ regulation: the role of GSK-3ß. Hypoxia. 2014 Apr 30;2:35-45.

49. Gjaltema RAF, Bank RA. Molecular insights into prolyl and lysyl hydroxylation of fibrillar collagens in health and disease. Crit Rev Biochem Mol Biol. 2017 Jan 2;52(1):74-95.

50. Cox TR, Erler JT. Remodeling and homeostasis of the extracellular matrix: implications for fibrotic diseases and cancer. Dis Model Mech. 2011 Mar;4(2):165-78.

51. Gilkes DM, Bajpai S, Wong CC, Chaturvedi P, Hubbi ME, Wirtz D, et al. Procollagen lysyl hydroxylase 2 is essential for hypoxia-induced breast cancer metastasis. Mol Cancer Res MCR. 2013 May;11(5):456-66.

52. Hofbauer K-H, Gess B, Lohaus C, Meyer HE, Katschinski D, Kurtz A. Oxygen tension regulates the expression of a group of procollagen hydroxylases. Eur J Biochem. 2003 Nov;270(22):4515-22.

53. Ewald CY, Landis JN, Abate JP, Murphy CT, Blackwell TK. Dauer-independent insulin/IGF-1signalling implicates collagen remodelling in longevity. Nature. 2014 Dec 15;519(7541):nature14021. 
54. Joshi KK, Matlack TL, Rongo C. Dopamine signaling promotes the xenobiotic stress response and protein homeostasis. EMBO J. 2016 01;35(17):1885-901.

55. Kim H, Choi D, Suh MC. Cuticle ultrastructure, cuticular lipid composition, and gene expression in hypoxia-stressed Arabidopsis stems and leaves. Plant Cell Rep. 2017 Jun;36(6):815-27.

56. Beloribi-Djefaflia S, Vasseur S, Guillaumond F. Lipid metabolic reprogramming in cancer cells. Oncogenesis. 2016 Jan 25;5(1):oncsis201549.

57. Katoh M, Nakagama H. FGF receptors: cancer biology and therapeutics. Med Res Rev. 2014 Mar;34(2):280-300.

58. Mulligan LM. RET revisited: expanding the oncogenic portfolio. Nat Rev Cancer. 2014 Mar;14(3):173-86.

59. Lampi MC, Reinhart-King CA. Targeting extracellular matrix stiffness to attenuate disease: From molecular mechanisms to clinical trials. Sci Transl Med. 2018 Jan 3;10(422).

60. Brenner S. The genetics of Caenorhabditis elegans. Genetics. 1974 May;77(1):71-94.

61. Davis MW, Hammarlund M, Harrach $T$, Hullett $P$, Olsen $S$, Jorgensen EM. Rapid single nucleotide polymorphism mapping in C. elegans. BMC Genomics. 2005 Sep 12;6:118.

62. Kamath RS, Ahringer J. Genome-wide RNAi screening in Caenorhabditis elegans. Methods San Diego Calif. 2003 Aug;30(4):313-21.

63. Mello CC, Kramer JM, Stinchcomb D, Ambros V. Efficient gene transfer in C.elegans: extrachromosomal maintenance and integration of transforming sequences. EMBO J. 1991 Dec;10(12):3959-70.

64. Schmieder R, Edwards R. Quality control and preprocessing of metagenomic datasets. Bioinforma Oxf Engl. 2011 Mar 15;27(6):863-4.

65. Kim D, Langmead B, Salzberg SL. HISAT: a fast spliced aligner with low memory requirements. Nat Methods. 2015 Apr;12(4):357-60.

66. Liao Y, Smyth GK, Shi W. featureCounts: an efficient general purpose program for assigning sequence reads to genomic features. Bioinforma Oxf Engl. 2014 Apr 1;30(7):923-30.

67. Love MI, Huber W, Anders S. Moderated estimation of fold change and dispersion for RNA-seq data with DESeq2. Genome Biol. 2014;15(12):550.

68. Maere S, Heymans K, Kuiper M. BiNGO: a Cytoscape plugin to assess overrepresentation of gene ontology categories in biological networks. Bioinforma Oxf Engl. 2005 Aug 15;21(16):3448-9.

69. Bindea G, Mlecnik B, Hackl H, Charoentong P, Tosolini M, Kirilovsky A, et al. ClueGO: a Cytoscape plug-in to decipher functionally grouped gene ontology and pathway annotation networks. Bioinforma Oxf Engl. 2009 Apr 15;25(8):1091-3. 
70. Thorvaldsdóttir H, Robinson JT, Mesirov JP. Integrative Genomics Viewer (IGV): high-performance genomics data visualization and exploration. Brief Bioinform. 2013 Mar;14(2):178-92.

\section{ACKNOWLEDGMENTS}

We thank the Caenorhabditis Genetics Center, National BioResource Project in Japan and the Million Mutation Project for C. elegans strains. We thank Bingying Wang and Eric Chuang for technical assistance, and Masako Asahina and Neel Singhal for discussion.

\section{FUNDING}

The work was supported by NIH grants R01GM117461, R00HL116654, ADA grant 1-16-IBS197, Pew Scholar Award, Alfred P. Sloan Foundation Fellowship, and Packard Fellowship in Science and Engineering (D.K.M) and Larry L. Hillblom start-up grant (D.K.M.) and fellowship (R.V).

\section{AUTHOR CONTRIBUTIONS}

R.V. and D.M. designed, performed and analyzed the experiments and wrote the manuscript. Y.L. performed RNA sequencing and bioinformatic analysis. D.M. supervised the project.

\section{COMPETING INTERESTS}

The authors declare that they have no competing financial interests.

\section{DATA AND MATERIALS AVAILABILITY}

The RNA-seq data have been deposited to X. All other data needed to evaluate the conclusions in the paper are present in the paper or the Supplementary Materials.

\section{FIGURE LEGENDS}


Fig. 1. comt-5p::GFP is upregulated by hypoxia in a hif-1-independent manner. A)

Fluorescent images of WT and hif-1(ia04) mutants carrying the genome-integrated dmals1 [comt-5p::GFP] transgene, exposed to normoxia or hypoxia. Representative of $>100$ animals B) Enlarged gray-scale images of $C$. elegans carrying comt-5p::GFP under normoxia and hypoxia. Arrows in normoxia image indicate head neurons with GFP signal. Arrowheads in hypoxia image indicate hypodermal cells and intestine with GFP signal. Representative of $>100$ animals C) Schematic diagram of the dmals 1 transgene. 2010 bp of upstream sequence of the comt-5 gene was cloned before the GFP-coding sequence followed by 3'UTR of the unc-54. The dmals 1 transgene also carries unc-54p::mCherry as an additional marker of expression. D) Analysis of the GFP signal by Western blot analysis of 20 randomly picked transgenic animals carrying the dmals1 [comt-5p::GFP] transgene. Histone $\mathrm{H} 3$ was used as a loading control. Transgenic animals express a cryptic nonspecific protein recognized by the GFP antibody (33 $\mathrm{kDa}$ ) that does not contribute to fluorescence in living animals. Representative of 3 independent experiments. E) qRT-PCR analysis of comt-5 expression in animals exposed to hypoxia (1\% $\left.\mathrm{O}_{2}\right)$, anoxia $\left(0 \% \mathrm{O}_{2}\right)$, or hypoxia-mimicking conditions $\left(\mathrm{CoCl}_{2}, \mathrm{NaSH}, \mathrm{KCN}\right) \cdot \mathrm{n} \geq 200$ total animals for each group with 3 independent biological replicates; ${ }^{* *}$ indicates $P<0.001$. F) qRTPCR analysis of comt-5 expression in normoxia- or hypoxia-exposed WT and hif-1(ia04) animals. $n \geq 200$ total animals for each group with 3 independent biological replicates; *** indicates $P<0.001$. Scale bars: $300 \mu \mathrm{m}$.

\section{Fig. 2. HIR-1 is a cell-autonomous regulator of signaling responses to hypoxia. A)}

Fluorescent images of transgenic animals carrying comt-5p::GFP transgene with various hir-1 alleles. Representative of $>100$ animals. B) Schematic diagram of hir-1 with indicated LOF alleles isolated from EMS mutagenesis (dma51), CRIPSR-Cas9 (dma101) and UV mutagenesis (tm3911 and tm4098). C) Schematic map of various hir-1 transgenes. D) Schematic representing domain arrangement in HIR-1. E) Fluorescent image of an animal carrying the 
transcriptional reporter hir-1p::GFP. Arrows indicate GFP signals in the hypoderm and seam cells. Representative of $>100$ animals. F) Fluorescent images of transgenic animals expressing comt-5p::GFP (worm 1) with hir-1(dma101) (worm 2) and hir-1(dma101); hir-1(+) array $d m E x 113$ (worm 3$) ;(n=100) .23 \%$ of animals found to be rescued by dmaEx113. Animals carrying extrachromosomal transgene $d m a E x 113$ [dpy-7p::hir-1;myo-2::mCherry] have mCherry fluorescence in the pharynx. G) Fluorescent images of transgenic animals carrying translational reporter of hir-1 [rpl-28p::hir-1sig::GFP::hir-1cod; myo-2p::mCherry]. Arrows show aggregated GFP signal. The transgenic marker myo-2p::mCherry expressed in the pharyngeal muscles is also shown. $\mathrm{n} \geq 10$ total animals for each group with $\mathrm{N} \geq 3$ independent biological replicates. H) SDS-PAGE (in the presense or absence of the reducing agent $\beta$-mercaptoethanol) and western blot analysis of crude extracts of transgenic animals expressing HIR-1::GFP. The star indicates high-molecular weight species of GFP::HIR-1 and the hash marks indicate processed GFP::HIR-1. Representative of 3 independent experiments. Scale bars: $300 \mu \mathrm{m}$.

\section{Fig. 3. Deficiency of specific cuticular genes mimics hypoxia-induced comt-5p::GFP}

activation. A) Fluorescent images of comt-5p::GFP expression in the hypoderm of wild-type animals or mutants lacking $d p y-2, d p y-3$ or perl-1. Representative of $>100$ animals. B) Schematic gene structures of $d p y-2, d p y-3$ and perl-1 showing the positions of EMS-derived LOF alleles. C) Protein domain organization of DPY-2, DPY-3 and PERL-1 with * indicating the positions of EMS-derived mutations. The G162R mutation in DPY-3 is in the conserved collagen domain, and the Q4R mutation in DPY-2 is in the signal peptide. The missense mutations in the PERL-1 are in the N-terminal peroxidase-like domain. D) Western blot analysis of WT animals or animals expressing the DPY-3-FLAG translational reporter exposed to normoxia or hypoxia. Representative of 2 independent experiments. E) Fluorescent images of animals carrying the col-19::GFP translational reporter exposed to normoxia, hypoxia, or $d p y-18(-), d p y-3(-)$, perl-1(-) and RNAi directed against let-268. Representative of 10 animals. Scale bars: $25 \mu \mathrm{m}$. B) 
Fluorescent images of comt-5p::GFP expression in animals treated with control or RNAi against the indicated genes essential for cuticle integrity. Representative of $>100$ animals. Scale bars: $300 \mu \mathrm{m}$.

Fig. 4. HIR-1 regulation of comt-5p::GFP requires the transcription factors NHR-49 and MDT-15. A) Fluorescent images of transgenic animals carrying comt-5p::GFP, and LOF and GOF of the indicated genes. Representative of $>100$ animals. B) Schematic gene structures of nhr-49 and mdt-15 showing the position of EMS-derived mutations. C) Western blot analysis of comt-5p::GFP expression in hir-1, hir-1; hif-1, and hir-1; nhr-49 mutants. Representative of 2 independent experiments. D) Protein sequence alignment of animal proteins orthologous to NHR-49 showing that Gly ${ }^{33}$ in NHR-49 is fully conserved in orthologues including human hepatocyte nuclear factor 4 (HNF4). E) qRT-PCR analysis of endogenous comt-5 expression in WT animals or $n h r-49$ or mdt-15 mutants exposed to normoxia or hypoxia. $N \geq 200$ total animals for each group with 3 independent biological replicates; ${ }^{* * *}$ indicates $P<0.001$. Scale bars: 300 $\mu \mathrm{m}$. F) Fluorescent images of transgenic animals carrying rpl-28p::nhr-49::Venus showing hypodermal nuclei exposed to normoxia or hypoxia.

Fig. 5. LET-756 is potential lignaf for HIR-1 A) Protein sequence alignment of intracellular kinase domains in human RTKs orthologous to HIR-1 indicating that HIR-1 possesses a conserved ATP binding site designated by *, a substrate proton acceptor site designated by \#, nucleotide binding sites designated by lines underlining the sequences, and conserved autophosphorylated tyrosine/serine residues found to be modified in human orthologues are designated by $\diamond$ above the sequences. B) Fluorescent images of comt-5p::GFP animals showing GFP induction elicited by RNAi against let-756. 
Fig. 6. HIR-1 mediates ECM homeostasis and anoxia resistance. A) qRT-PCR analysis of comt-5 expression in WT, hir-1(dma101), hir-1(dma101); hif-1(ia04) and hir-1(dma101); nhr49(nr2041) mutant animals. $N \geq 200$ total animals for each group with 3 independent biological replicates; ${ }^{* * *}$ indicates $P<0.001$. B) Fluorescent images of animals expressing the col19::GFP translational reporter in the presence or absence of hir-1 RNAi. Representative of 10 animals. C) Cuticle integrity as detected by nuclear staining with Hoechst 22358 in the hypoderm of WT or hir-1(dma101) animals. Numbers represent percentages of animals with stained nuclei ( $n=10$ animals). D) Representative plot showing the average locomotion speed of a WT or hir-1 worm in a 10-minute time frame recorded after 24 hours of re-oxygenation. E) Plot showing representative locomotor tracks of WT and hir-1 animals in a 10-minute time frame recorded after 24 hours of re-oxygenation. Position is detected based on the mid-point of the worm. F) Displacement of WT and hir-1 mutants in a 10-minute time frame recorded before exposure to hypoxia, and after 0 - $10 \mathrm{~min}, 2$ and 24 hours of re-oxygenation; ${ }^{* * *}$ indicates $\mathrm{P}<$ 0.001. $\mathrm{N}=10$ animals for each group with 3 independent biological replicates. G) Schematic model of the proposed HIR-1 pathway that mediates cellular response to hypoxia-induced ECM remodeling to maintain ECM homeostasis in C. elegans. Inactive components are shown in gray and activated components are shown in black. Scale bars: $25 \mu \mathrm{m}$.

Table 1. EMS-derived mutants with constitutive comt-5p::GFP expression.

\begin{tabular}{|c|c|c|c|c|c|c|}
\hline Strain & Allele & Sequence & Gene & Homology & Localization & Penetrance \\
\hline DMS99 & dma11 & Unknown & Unknown & Unknown & Unknown & $100 \%(210)$ \\
\hline DMS176 & dma51 & C24G6.2 & hir-1 & tyrosine & plasma & $82 \%(134)$ \\
\hline DMS177 & dma52 & T14B4.6 & $d p y-2$ & collagen & extracellular & $92 \%(145)$ \\
\hline DMS127 & dma22 & EGAP7.1 & $d p y-3$ & collagen & plasma & $75 \%(128)$ \\
\hline DMS180 & dma53 & C46A5.4 & perl-1 & animal & extracellular & $88 \%(105)$ \\
\hline
\end{tabular}


bioRxiv preprint doi: https://doi.org/10.1101/302638; this version posted April 16, 2018. The copyright holder for this preprint (which was not certified by peer review) is the author/funder, who has granted bioRxiv a license to display the preprint in perpetuity. It is made available under aCC-BY 4.0 International license.

FIG. 1

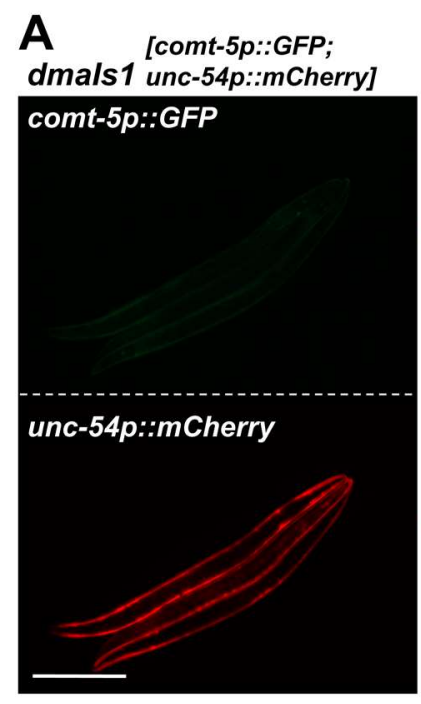

B

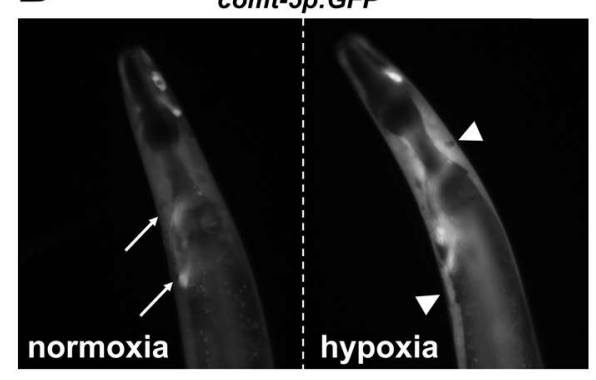

D

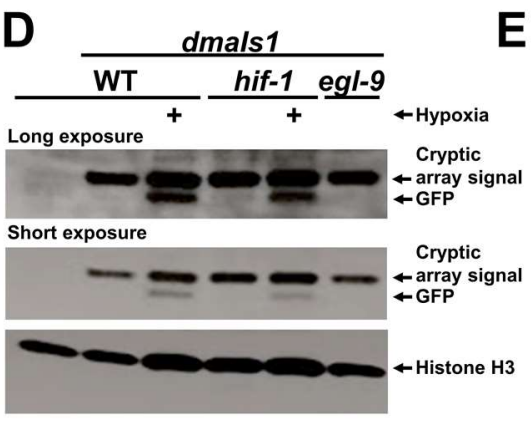

dmals1 + hypoxia

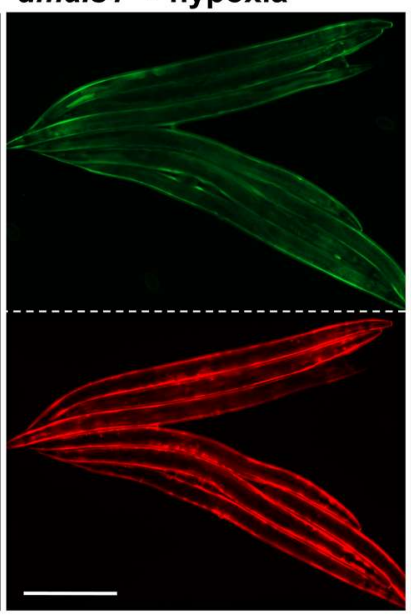

C

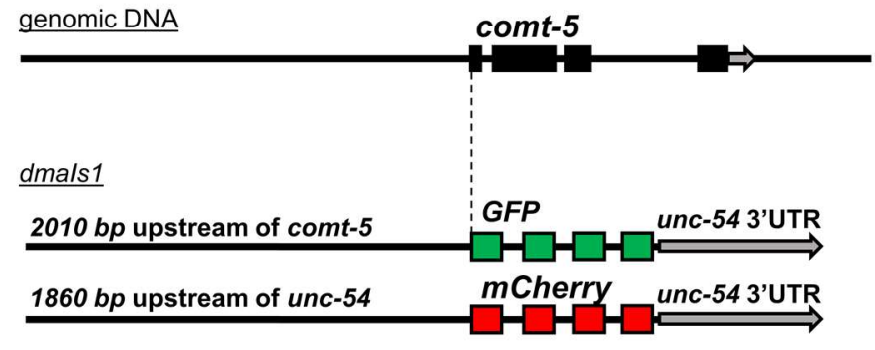

$\mathbf{F}$
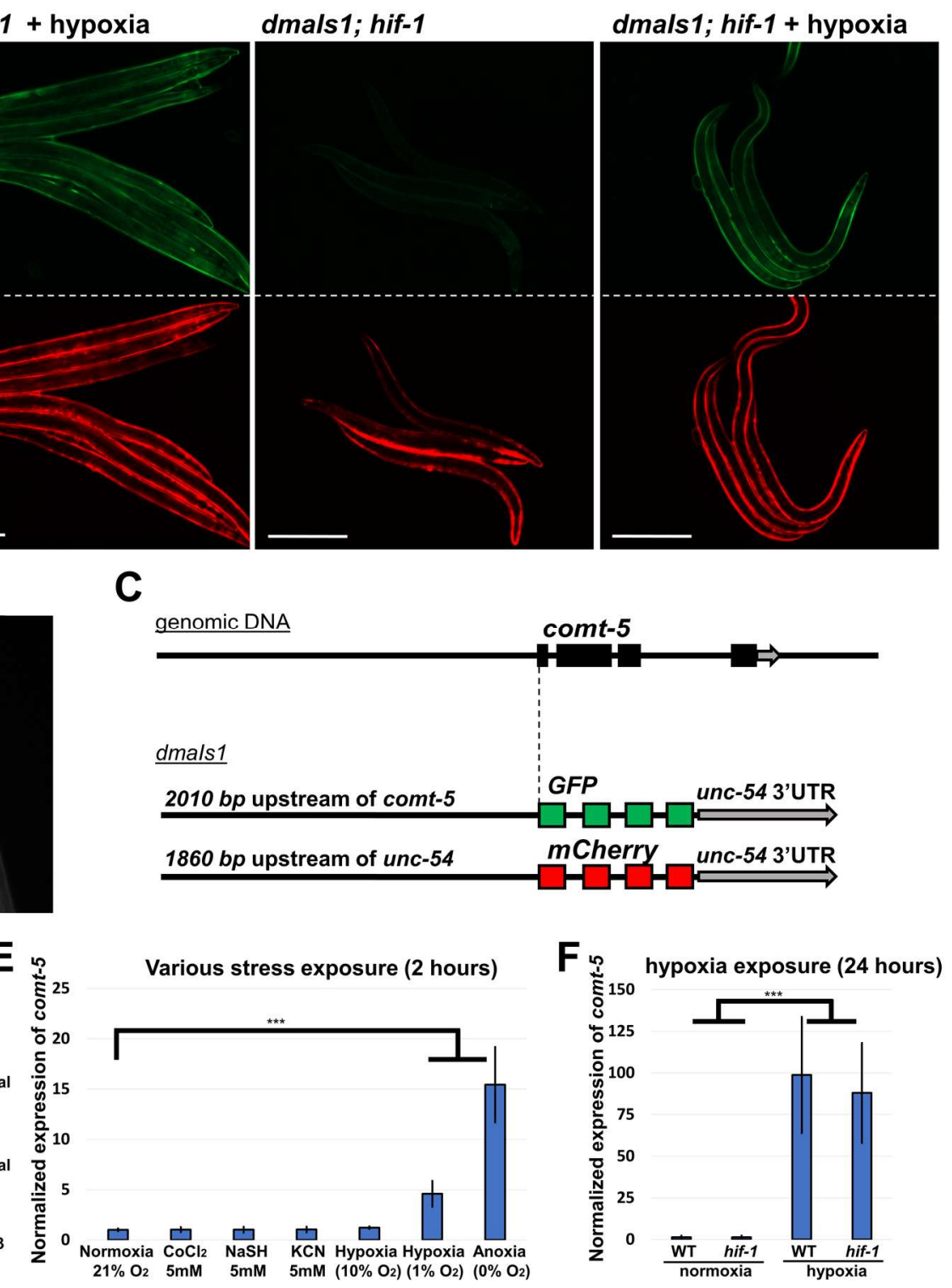
bioRxiv preprint doi: https://doi.org/10.1101/302638; this version posted April 16, 2018. The copyright holder for this preprint (which was not certified by peer review) is the author/funder, who has granted bioRxiv a license to display the preprint in perpetuity. It is made available under aCC-BY 4.0 International license.

FIG. 2

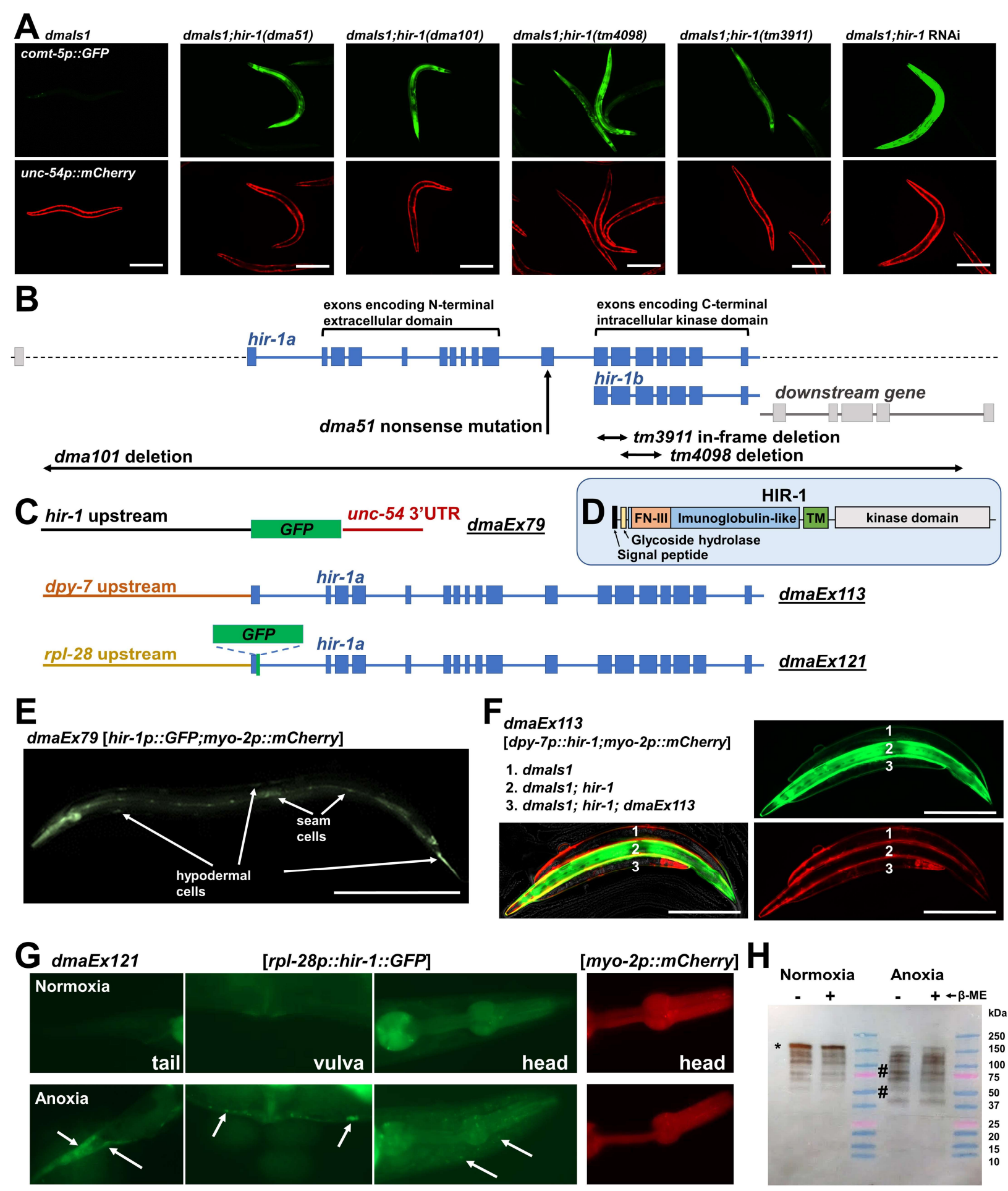


bioRxiv preprint doi: https://doi.org/10.1101/302638; this version posted April 16, 2018. The copyright holder for this preprint (which was not certified by peer review) is the author/funder, who has granted bioRxiv a license to display the preprint in perpetuity. It is made available under aCC-BY 4.0 International license.

FIG. 3
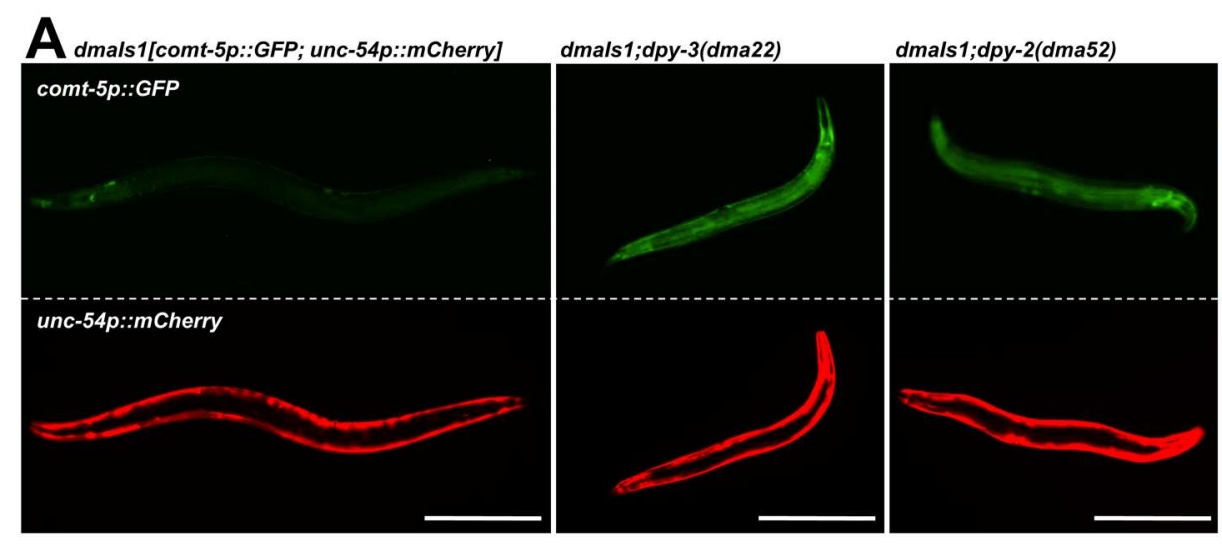

dmals1;perl-1(dma236)

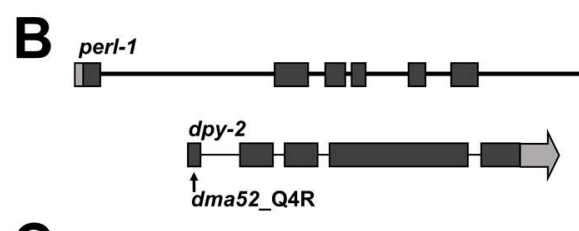

C
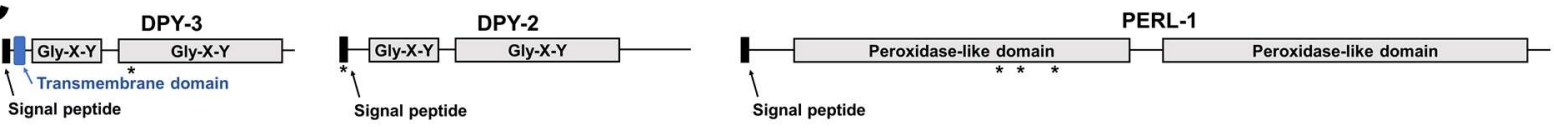

D

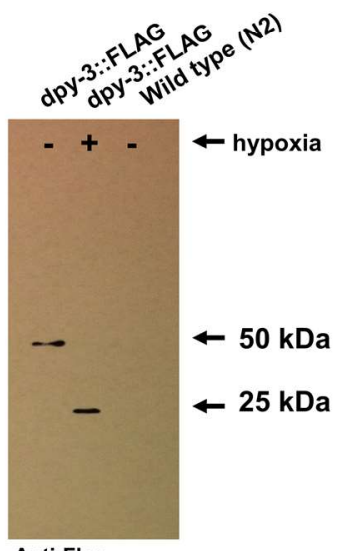

Anti-Flag
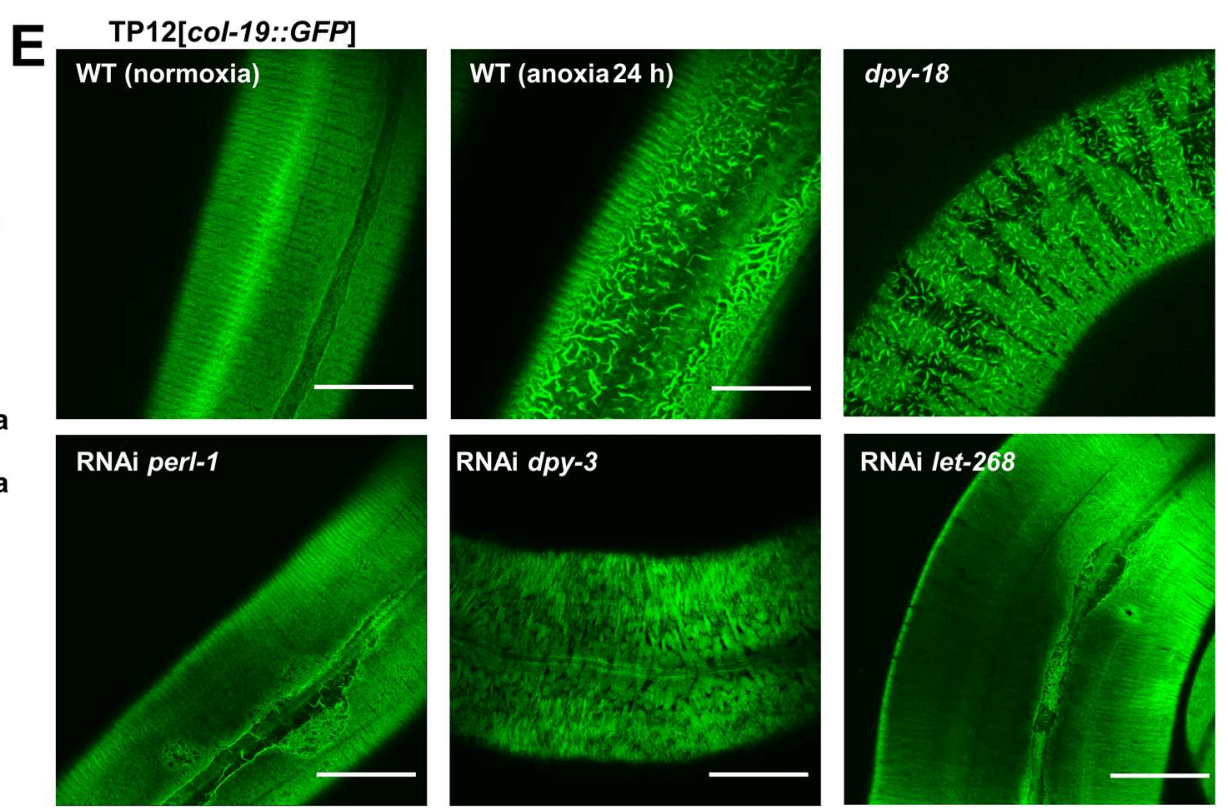
bioRxiv preprint doi: https://doi.org/10.1101/302638; this version posted April 16, 2018. The copyright holder for this preprint (which was not certified by peer review) is the author/funder, who has granted bioRxiv a license to display the preprint in perpetuity. It is made available under aCC-BY 4.0 International license.

FIG. 4

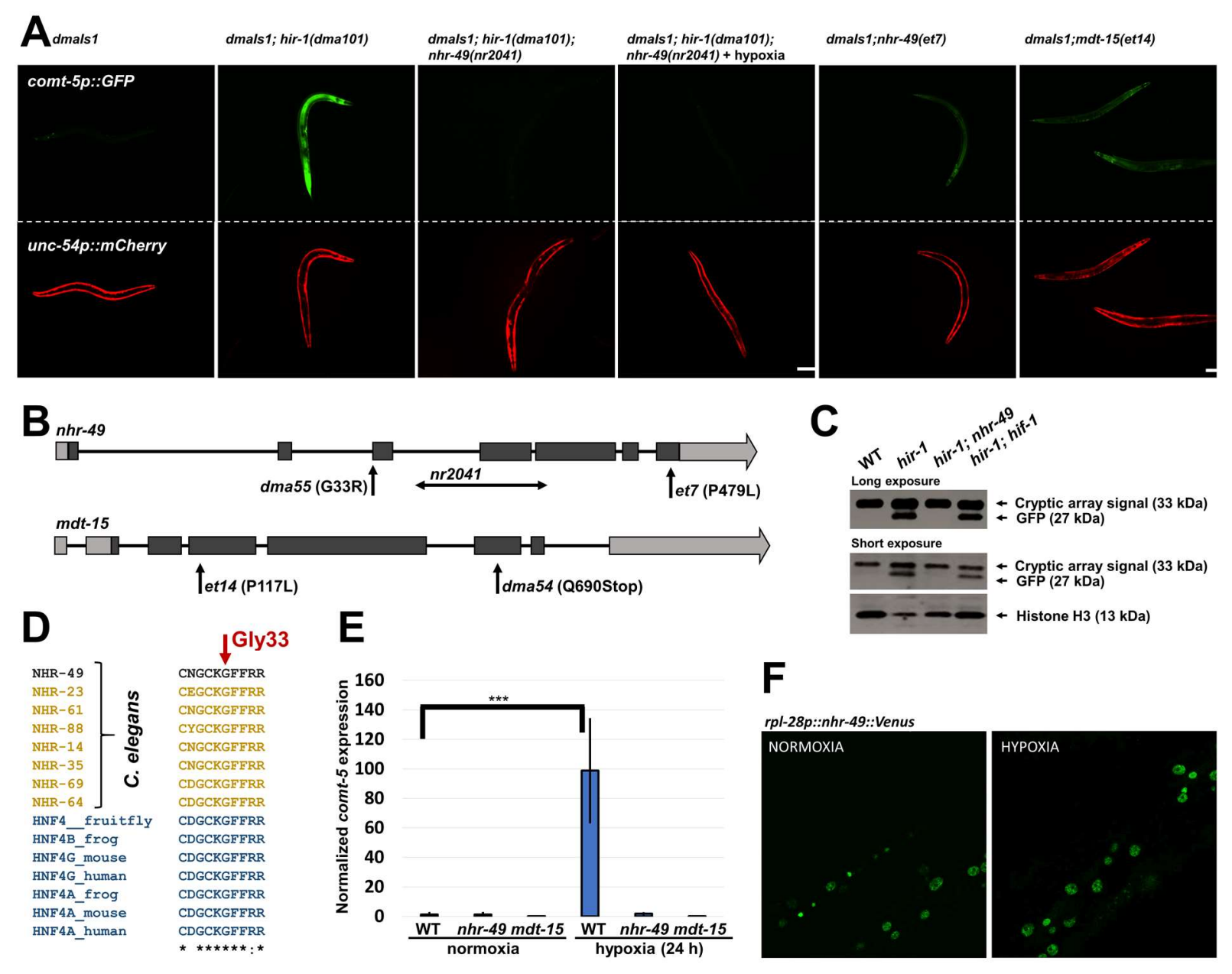


bioRxiv preprint doi: https://doi.org/10.1101/302638; this version posted April 16, 2018. The copyright holder for this preprint (which was not certified by peer review) is the author/funder, who has granted bioRxiv a license to display the preprint in perpetuity. It is made available under aCC-BY 4.0 International license.

FIG. 5

A

FKFQME--QVGKNNMNRY--_ VSY------SS--SGARR---------PSLDSMENQVSVDAFK ILEDPKWEEPRKNLVL--GKTLGEGEFGKVVKATAFHLKGR-----------AGYTTVAVKMLKENASPSELR LARQESLE--SGSSGKSSSSLVRGVR--LSSSGPALLAGLVSLDLPLDPLWE FPRDRLVL--GKPLGEGCEGQVVRAEAFGMDPAR----------PDQASTVAVKMLKDNASDKDLA LKRQVS--LESNASMSSNTPLVRIAR--LSSGEGPTLANVSELELPADPKWELSRARLTL--GKPLGEGCEGQVVMAEAIGIDKDR--_------AAKPVTVAVKMLKDDATDKDLS LRRQVTVSADSSASMNSGVLLVRPSR--LSSSGTPMLAGVSEYELPEDPRWELPRDRLVL--GKPLGEGCEGQVVLAEAIGLDKDK---------PNRVTKVAVKMLKSDATEKDLS

EFLREIALMKTLGYHERLVNMLACVTESEPLCLVVEYCDNGDLLKFLRERCKYMMKLDDLGINYHDP PENENYDTNMIVTLKQLLQFAVQISYGLEYLSQKGEVHRDVAARNVLVHEGTA DLLSEFNVLKQVN-HPHVIKLYGACSQDGPLLLIVEYAKYGSLRGELRESRKVGPGYLGSGGSRNS--SSLDHPDERALTMGDI ISFAWQI SQGMQYLAEMKLVHRDLAARNI LVAEGRK DLVSEMEVMKLIGRHKN I INLLGVCTQEGPLYVIVECAAKGNLREFLRARRP PGPDLSPD--_-_---GPRSSEG PLSFPVLVSCAYQVARGMQYLESRKCIHRDLAARNVLVTEDNV DLVSEMEMMKMI GKHKN I INLLGACTQGGPLYVLVEYAAKGNLREELRARRPPGLDYSFD--_---TCKPPEEQLTFKDLVSCAYQVARGMEYLASQKCIHRDLAARNVLVTEDNV DLISEMEMMKMIGKHKNI INLLGACTQDGPLYVIVEYASKGNLREYLQARRP PGIEYCYN-_-_-_-PSHNPEEQLSSKDIVSCAYQVARGMEYLASKKCIHRDLAARNVLVTEDNV

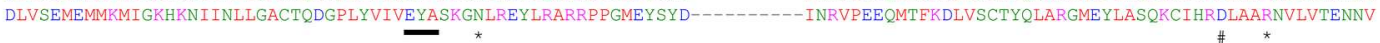

CKIGDFGLCRYIYADQSQYKSKGGKLPLKWMSPEAIRHYEFS IKSDIWSEGILLFEVITLGGSPYPGMP PEDVLPFLEGGGRIEKPDNCPENFYDVMMQCWNADPDDRIEFSDVRMQLAT MKISDFGLSRDVYEEDSYVKRSQGRIPVKWMAIESLFDHIYTTQSDVWSEGVLLWEIVTLGGNPY PGIPPERLFNLIKTGHRMERPDNCSEEMYRLMLQCWKQEPDKRPVVADISKDLEKK

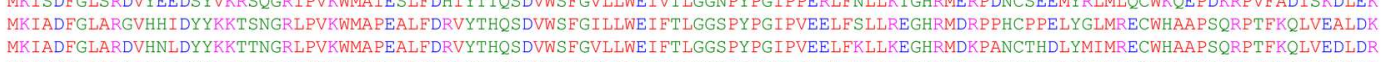

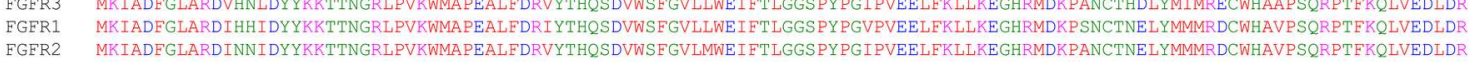

HIR-1 QLEDITEDYSYLKLDAAKDYYNYOYGDEKKTDVVIIPDEIIKPSKLIMDDIS---------EKNLVIEQ

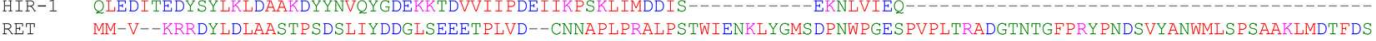
FGFR4 MIM-VER

FGFR4

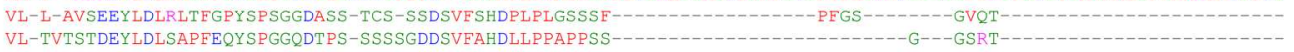

IV-ALTSNQEYLDLSMPLDQYSPSEPDTRSSTCSSGEDSVFSHEPLPEEPCLP----19 -RHPAQIANG---GLKRR--10

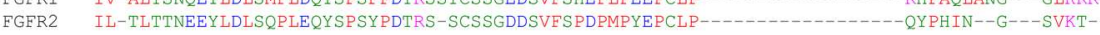

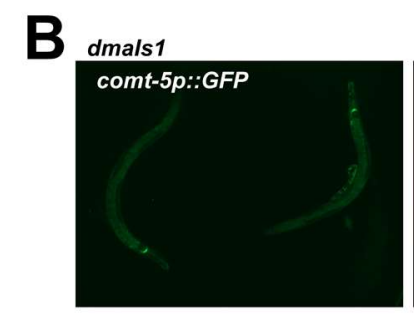

dmals1; RNAi let-756

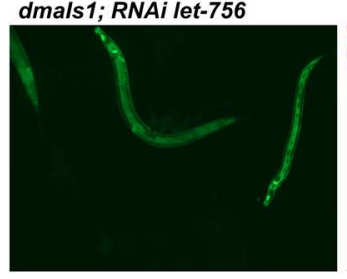

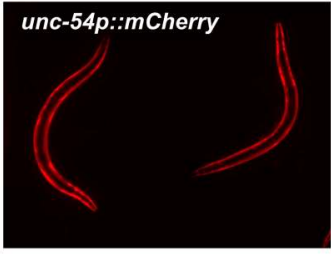

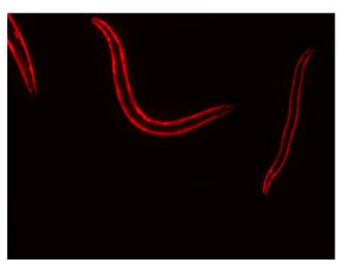

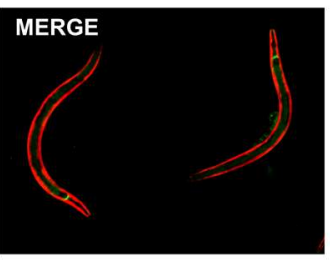

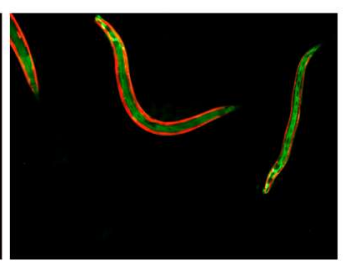

C

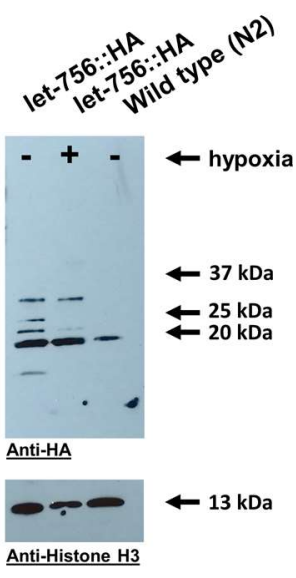


FIG. 6

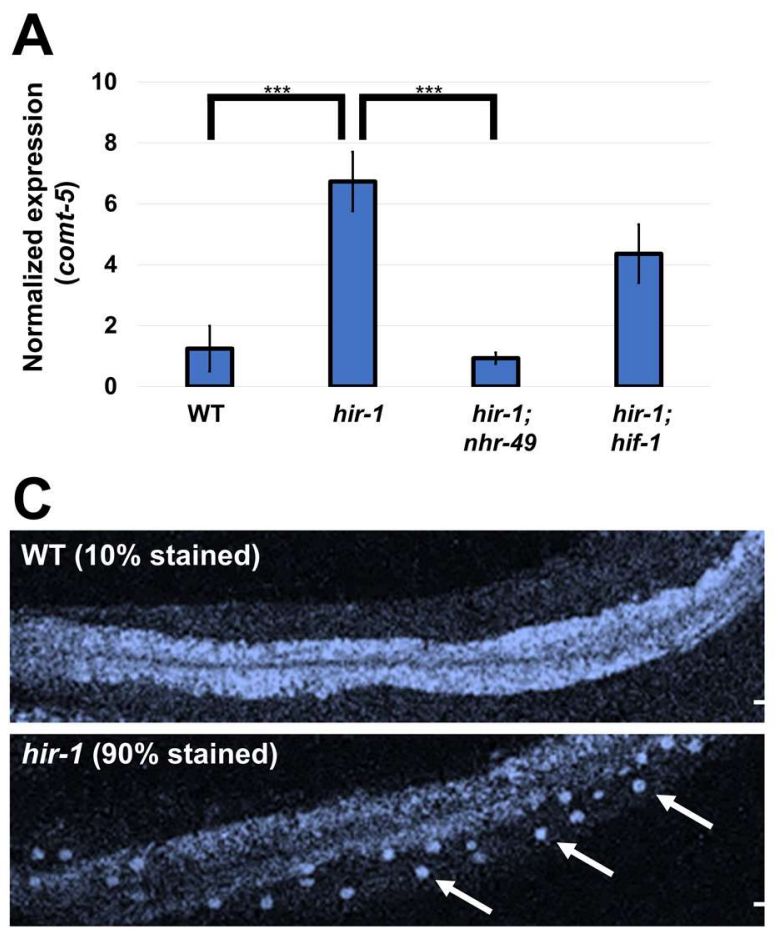

B
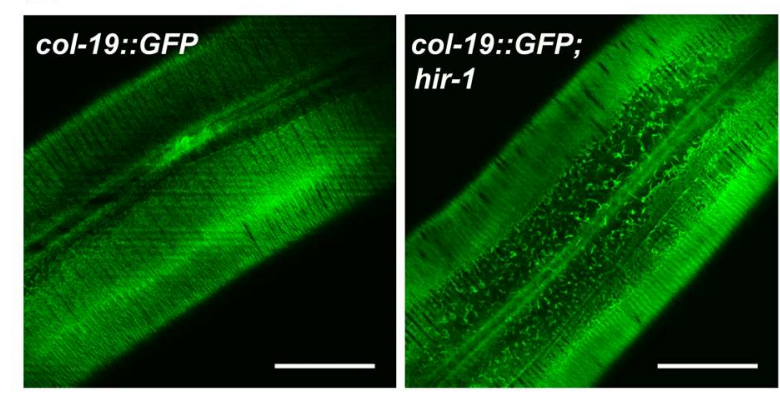

\section{D}
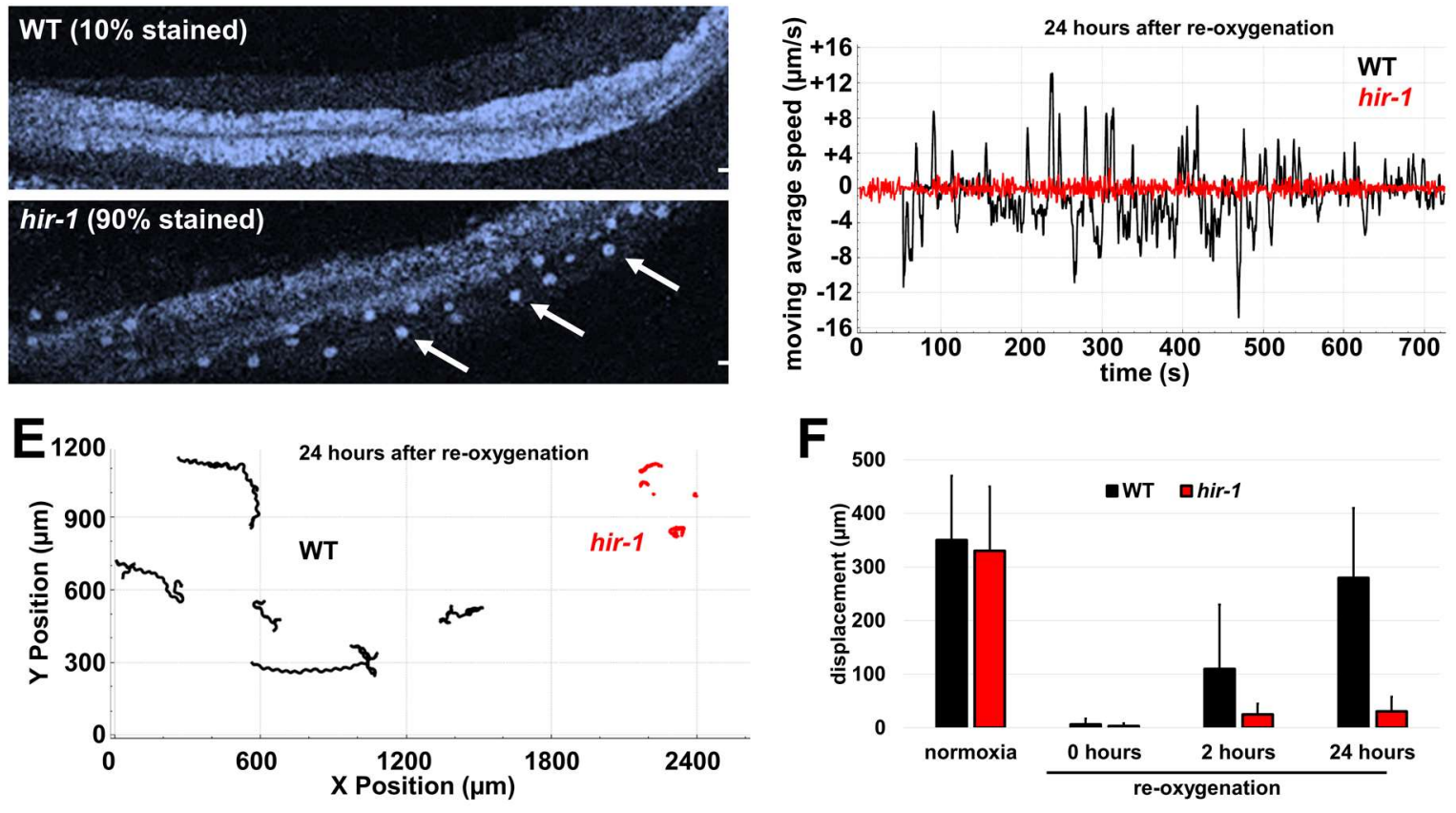

G
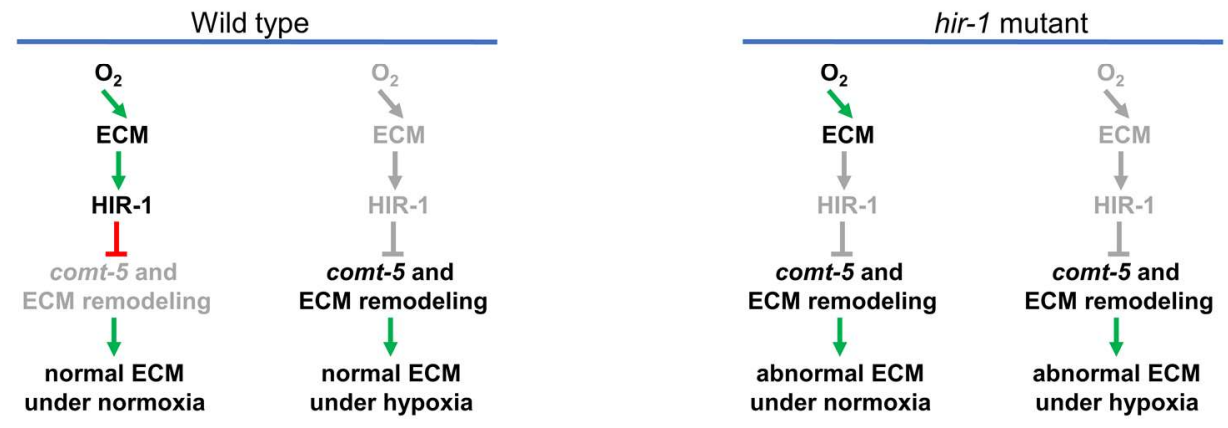